\title{
Aryloxy Alkyl Magnesium versus Dialkyl Magnesium in the Lanthanidocene Catalyzed Coordinative Chain Transfer Polymerization of Ethylene
}

\author{
Mikhail E. Minyaev, ${ }^{\dagger}$ Pavel D. Komarov ${ }^{\dagger}$ Dmitrii M. Roitershtein $,{ }^{*},{ }^{\dagger}, 8$ Konstantin A. Lyssenko, \\ Ilya E. Nifant'ev, ${ }^{*},{ }^{\dagger}, /$ Lada N. Puntus, ${ }^{\dagger,}$ Evgenia A. Varaksina, ${ }^{\dagger, \|}$ Roman S. Borisov, ${ }^{\dagger, \triangle}$ Viktor P. \\ Dyadchenko," Pavel V. Ivchenko ${ }^{\dagger, / /}$ \\ $\dagger$ A.V. Topchiev Institute of Petrochemical Synthesis, Leninsky pr. 29, Moscow 119991, Russian Federation. \\ N.D. Zelinsky Institute of Organic Chemistry, Russian Academy of Sciences, Leninsky pr. 47, Moscow 119991, \\ Russian Federation. \\ $\S$ National Research University Higher School of Economics, Miasnitskaya Str. 20, Moscow 101000, Russian \\ Federation. \\ $\perp$ G.V. Plekhanov Russian University of Economics, 36 Stremyanny Per., Moscow 117997, Russian Federation. \\ // M.V. Lomonosov Moscow State University, Chemistry Department, 1 Leninskie Gory Str., Building 3, Moscow \\ 119991, Russian Federation. \\ g V.A. Kotel'nikov Institute of Radioengineering and Electronics, Russian Academy of Sciences, 11-7 Mokhovaya \\ Str., Moscow 125009, Russian Federation. \\ \# P.N. Lebedev Physical Institute, Russian Academy of Sciences, 53 Leninsky Prospect, Moscow 119991, Russian \\ Federation. \\ $\triangle$ Peoples' Friendship University of Russia, 6 Miklukho-Maklaya Str., Moscow 117198, Russian Federation. \\ *Corresponding Authors: D.M.R. roiter@yandex.ru, I.E.N. ilnif@yahoo.com
}

\section{Supporting Information}

S1. General experimental remarks

S2

S2. Preparation of lanthanidocenes

S3-S6

S2.1. NMR spectra of triarylcyclopentadienes

S3-S5

S2.2. Synthesis of complexes

S6-S8

S3. X-ray structure determination

S9-S18

S3.1. X-ray diffraction studies, crystallographic data and refinement details

S9-S10

S3.2. $\left[\left(\eta^{5}-1,2,4-\mathrm{Ph}_{3}-\mathrm{C}_{5} \mathrm{H}_{2}\right)_{2} \mathrm{NdCl}_{2} \mathrm{~K}(\mathrm{THF})_{2}\right]_{2}, \mathbf{N d 1}$

S11-S13

S3.3. $\left\{\left[\eta^{5}-1,2-\mathrm{Ph}_{2}-4-\left(4-\mathrm{MeOC}_{6} \mathrm{H}_{4}\right) \mathrm{C}_{5} \mathrm{H}_{2}\right]_{2} \mathrm{Nd}\left(\mu^{2}-\mathrm{Cl}\right)\left(\mu^{3}-\mathrm{Cl}\right) \mathrm{K}(\mathrm{THF})_{2}\right\}_{2}, \mathbf{N d} 2$

S3.4. $\left\{\left[\eta^{5}-1,2-\mathrm{Ph}_{2}-4-\left(2-\mathrm{MeOC}_{6} \mathrm{H}_{4}\right) \mathrm{C}_{5} \mathrm{H}_{2}\right]_{2} \mathrm{NdCl}_{2}\left[\mathrm{~K}(\mathrm{THF})_{4}\right]\right\}(\mathrm{THF})_{0.5}, \mathbf{N d 3}$

S15-S16

S3.5. $\left\{\left[\eta^{5}-1,2-\mathrm{Ph}_{2}-4-\left(2-\mathrm{MeOC}_{6} \mathrm{H}_{4}\right) \mathrm{C}_{5} \mathrm{H}_{2}\right]_{2} \mathrm{NdCl}\right\}, \mathbf{N d} 4$

S17-S18

S4. The studies of the CCTP of ethylene

S18-S25

S4.1. Oligomerization and derivatization

S18-S19

S4.2. NMR spectra the reaction products

S20-S21

S4.3. Characterization of [PE-DMAP]I

S22-S26

S5. Optical measurements

S27

S6. DFT calculations 


\section{S1. General experimental remarks}

All synthetic manipulations were carried out in a prepurified argon atmosphere or under vacuum in absolute solvent media using a glove box or standard Schlenk and vacuum line techniques. All glassware was oven-dried prior to use. Tetrahydrofuran (THF) and diethyl ether $\left(\mathrm{Et}_{2} \mathrm{O}\right)$ were distilled from sodium/benzophenone. $n$-Pentane, $n$-hexane and $n$-heptane were distilled from $\mathrm{Na} / \mathrm{K}$ alloy. Toluene was distilled from sodium/benzophenone in the presence of dibenzo-18crown-6. $\mathrm{Mg}^{n} \mathrm{Bu}_{2}$ (1.0 M solution in heptane, Sigma-Aldrich) and 2,6-di-tert-butyl-4-methylphenol (butylated hydroxytoluene BHT-H, 99\%, Sigma-Aldrich) were used as purchased. Ethylene (99.95\%, Linde, Moscow, Russian Federation) was additionally purified during the polymerization procedure by passing it through a column containing $4 \AA$ molecular sieves impregnated with $\mathrm{Na} / \mathrm{K}$ alloy and then through a column containing 3 mass $\% \mathrm{MnO}{ }^{1,2}$

1,2-Diphenyl-4-(2-methoxyphenyl)cyclopenta-1,3-diene, 1,2,4-triphenylcyclopenta-1,3diene and 1,2-diphenyl-4-(4-methoxyphenyl)cyclopenta-1,3-diene were obtained by the published method, ${ }^{3}$ recrystallized from methyl tert-butyl ether and vacuum sublimed prior to use (NMR spectra are provided in Figs. S1-S6). Compounds $\mathrm{TbCl}_{3}(\mathrm{THF})_{3}, \quad \mathrm{NdCl}_{3}(\mathrm{THF})_{2.5}{ }^{4}$ (BHT) $\mathrm{Mg}(\mathrm{THF}){ }_{2}{ }^{n} \mathrm{Bu},{ }^{5} \mathrm{PhCH}_{2} \mathrm{~K},{ }^{6,7}$ and $\left[\left(1,2,4-\mathrm{Ph}_{3} \mathrm{C}_{5} \mathrm{H}_{2}\right)_{2} \mathrm{TbCl}_{2} \mathrm{~K}\right]_{2}$ (Tb1) ${ }^{8}$ were prepared according to literature procedures. Complexes Nd1-Nd3 were synthesized according to the method described for related compounds, ${ }^{6,8,9}$ see $\mathrm{S} 2.2$.

THF- $\mathrm{d}_{8}\left(99.5\right.$ atom \% D, Sigma-Aldrich) and toluene- $\mathrm{d}_{8}$ (99.6 atom \% D, Sigma-Aldrich) were distilled from $\mathrm{Na} / \mathrm{K}$ alloy. $\mathrm{CDCl}_{3}(99.8$ atom \% D, Cambridge Isotope Laboratories, Inc., Cambridge, UK) was used as purchased. NMR spectra were recorded with Bruker AV400 (400 $\mathrm{MHz}$ for ${ }^{1} \mathrm{H}$ and $100.6 \mathrm{MHz}$ for ${ }^{13} \mathrm{C}$ ) or Bruker AV-600 (600 MHz for ${ }^{1} \mathrm{H}$ and $150.9 \mathrm{MHz}$ for ${ }^{13} \mathrm{C}$ ) spectrometers. MALDI-TOF mass spectra were recorded with a Bruker autoflex speed instrument in the positive-ion reflectron mode, using a solid-state UV-laser $(\lambda=355 \mathrm{~nm})$. Standard differential scanning calorimetry (DSC) thermograms were recorded on a DSC823 Mettler Toledo system at a rate of $10^{\circ} \mathrm{C} / \mathrm{min}$ under an argon atmosphere.

The lanthanide content was determined by direct complexometric titration with the disodium salt of ethylenediaminetetraacetic acid, using the xylenol orange indicator. Elemental analyses were performed with a PerkinElmer 2400 Series II elemental CHNS/O analyzer. 


\section{S2. Preparation of lanthanidocenes}

S2.1. NMR spectra of triarylcyclopentadienes

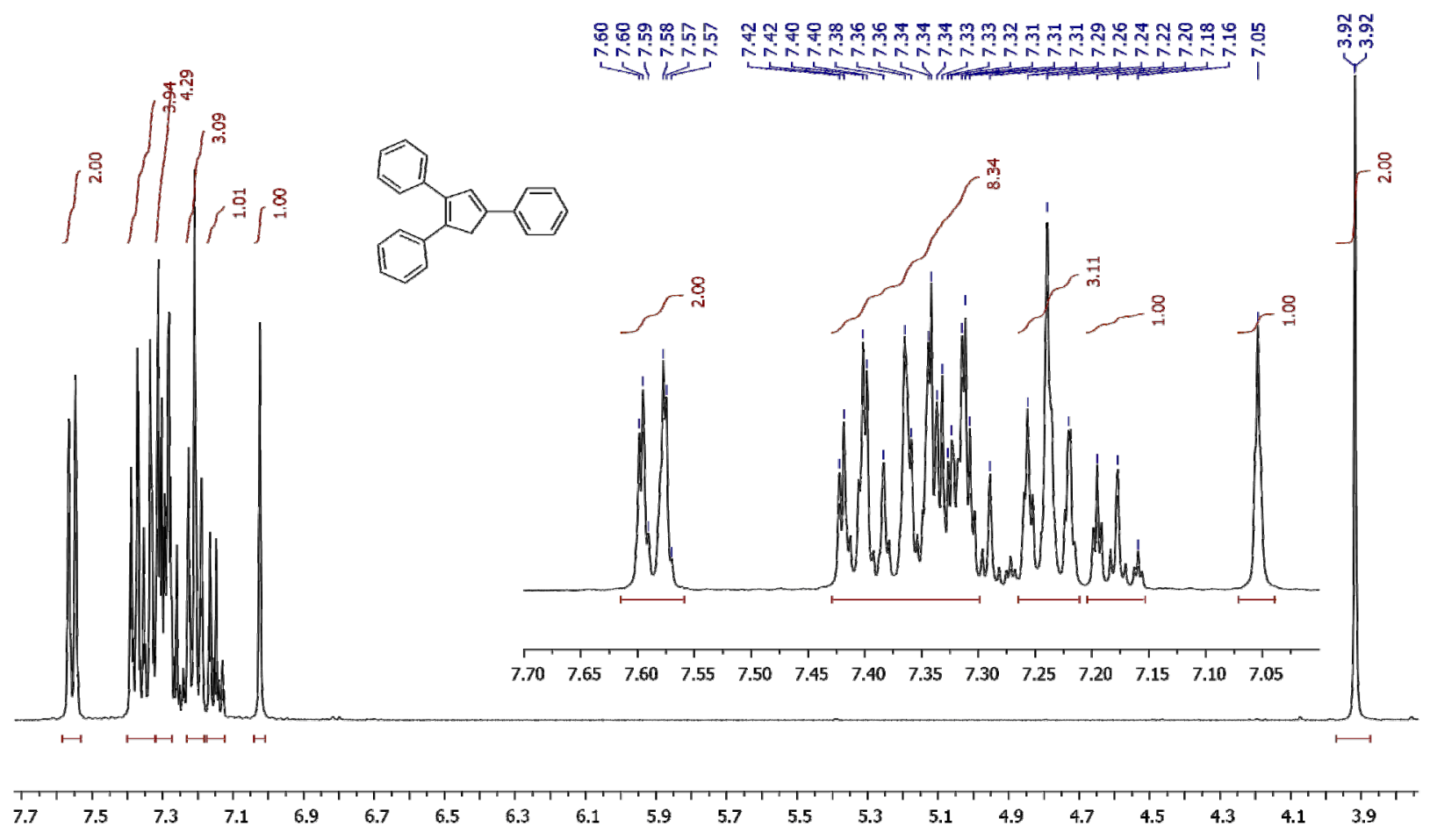

Fig. S1. ${ }^{1} \mathrm{H}$ NMR spectrum $\left(\mathrm{CDCl}_{3}, 400 \mathrm{MHz}, 20^{\circ} \mathrm{C}\right)$ of $1,2,4-\mathrm{Ph}_{3} \mathrm{C}_{5} \mathrm{H}_{3}$

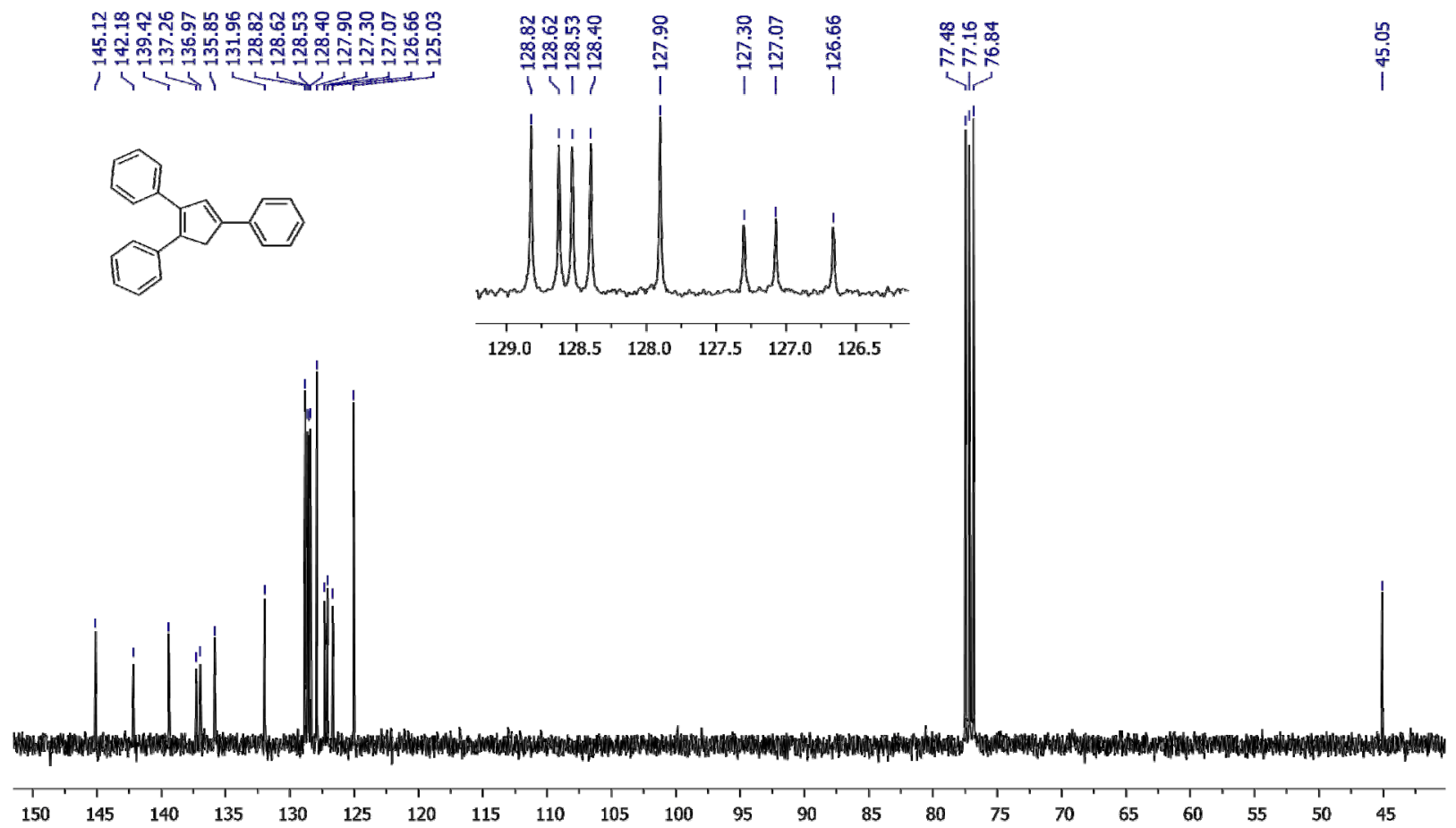

Fig. S2. ${ }^{13} \mathrm{C}\left\{{ }^{1} \mathrm{H}\right\}$ NMR spectrum $\left(\mathrm{CDCl}_{3}, 101 \mathrm{MHz}, 20^{\circ} \mathrm{C}\right)$ of $1,2,4-\mathrm{Ph}_{3} \mathrm{C}_{5} \mathrm{H}_{3}$ 




Fig. S3. ${ }^{1} \mathrm{H}$ NMR spectrum $\left(\mathrm{CDCl}_{3}, 600 \mathrm{MHz}, 20^{\circ} \mathrm{C}\right)$ of 1,2-Ph $-4-\left(4-\mathrm{MeOC}_{6} \mathrm{H}_{4}\right) \mathrm{C}_{5} \mathrm{H}_{3}$

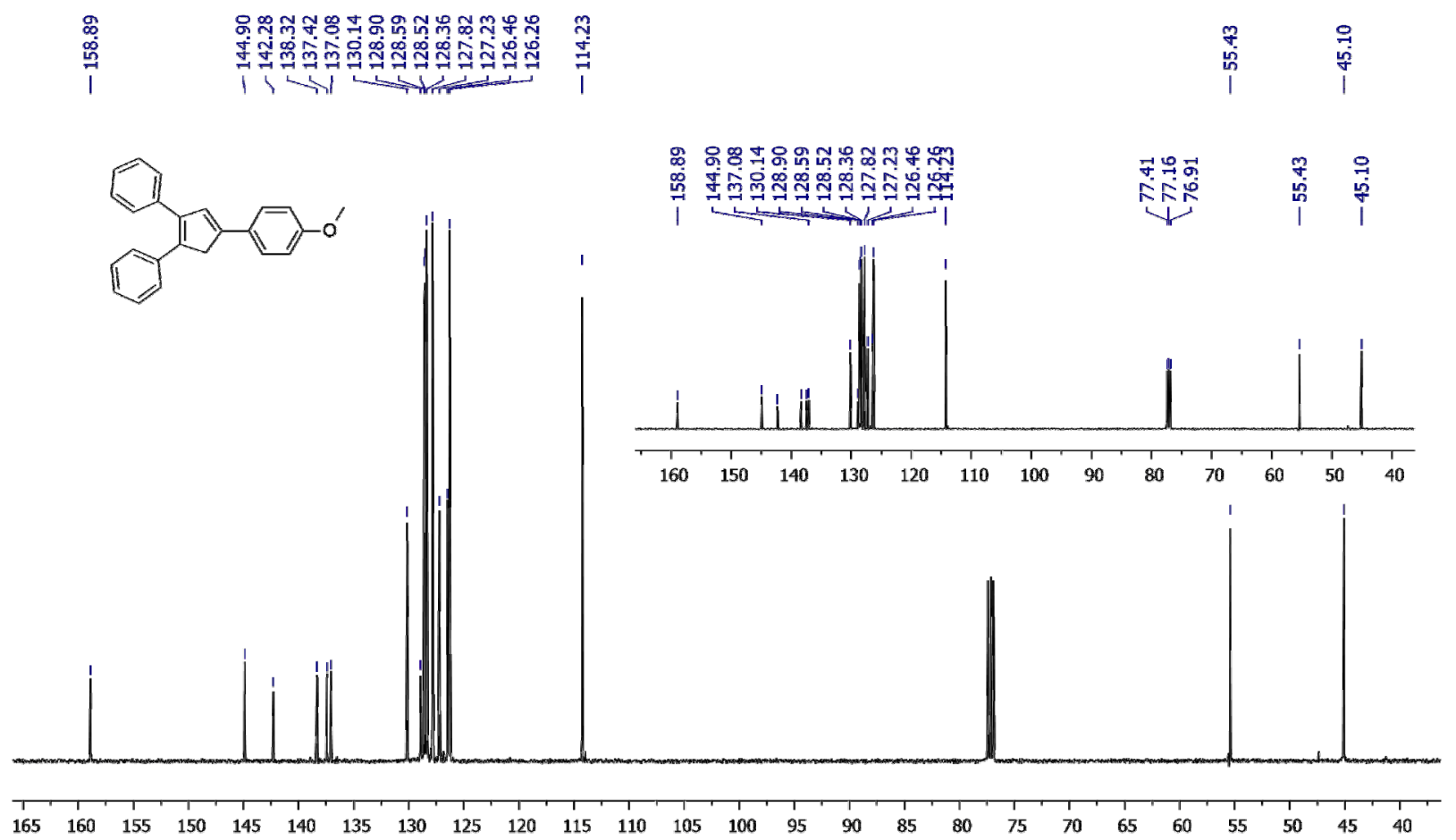

Fig. S4. $\left.{ }^{13} \mathrm{C}^{1} \mathrm{H}\right\}$ NMR spectrum $\left(\mathrm{CDCl}_{3}, 126 \mathrm{MHz}, 20^{\circ} \mathrm{C}\right)$ of 1,2-Ph $-4-\left(4-\mathrm{MeOC}_{6} \mathrm{H}_{4}\right) \mathrm{C}_{5} \mathrm{H}_{3}$ 


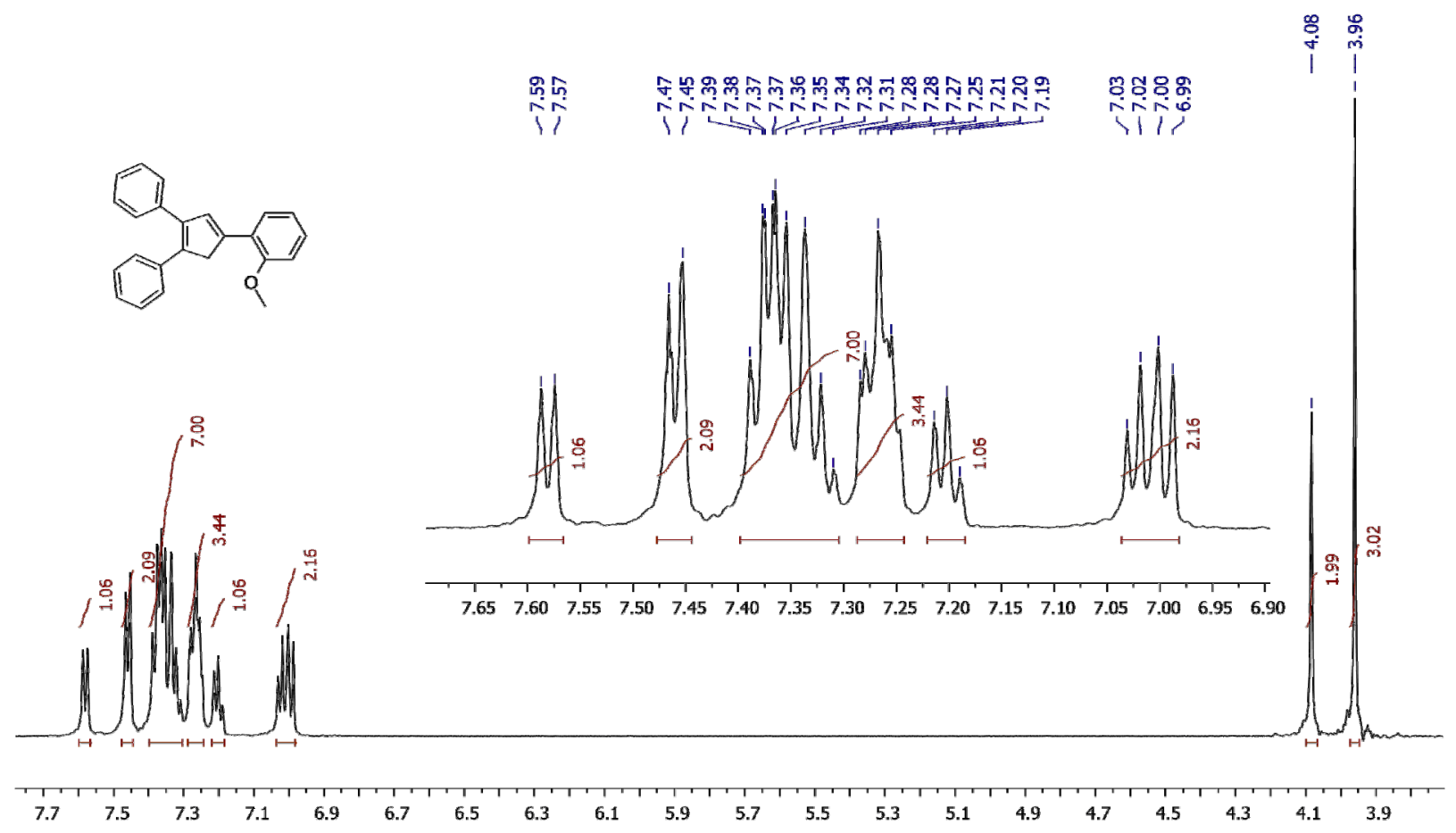

Fig. S5. ${ }^{1} \mathrm{H}$ NMR spectrum $\left(\mathrm{CDCl}_{3}, 600 \mathrm{MHz}, 20^{\circ} \mathrm{C}\right)$ of $1,2-\mathrm{Ph}_{2}-4-\left(2-\mathrm{MeOC}_{6} \mathrm{H}_{4}\right) \mathrm{C}_{5} \mathrm{H}_{3}$.

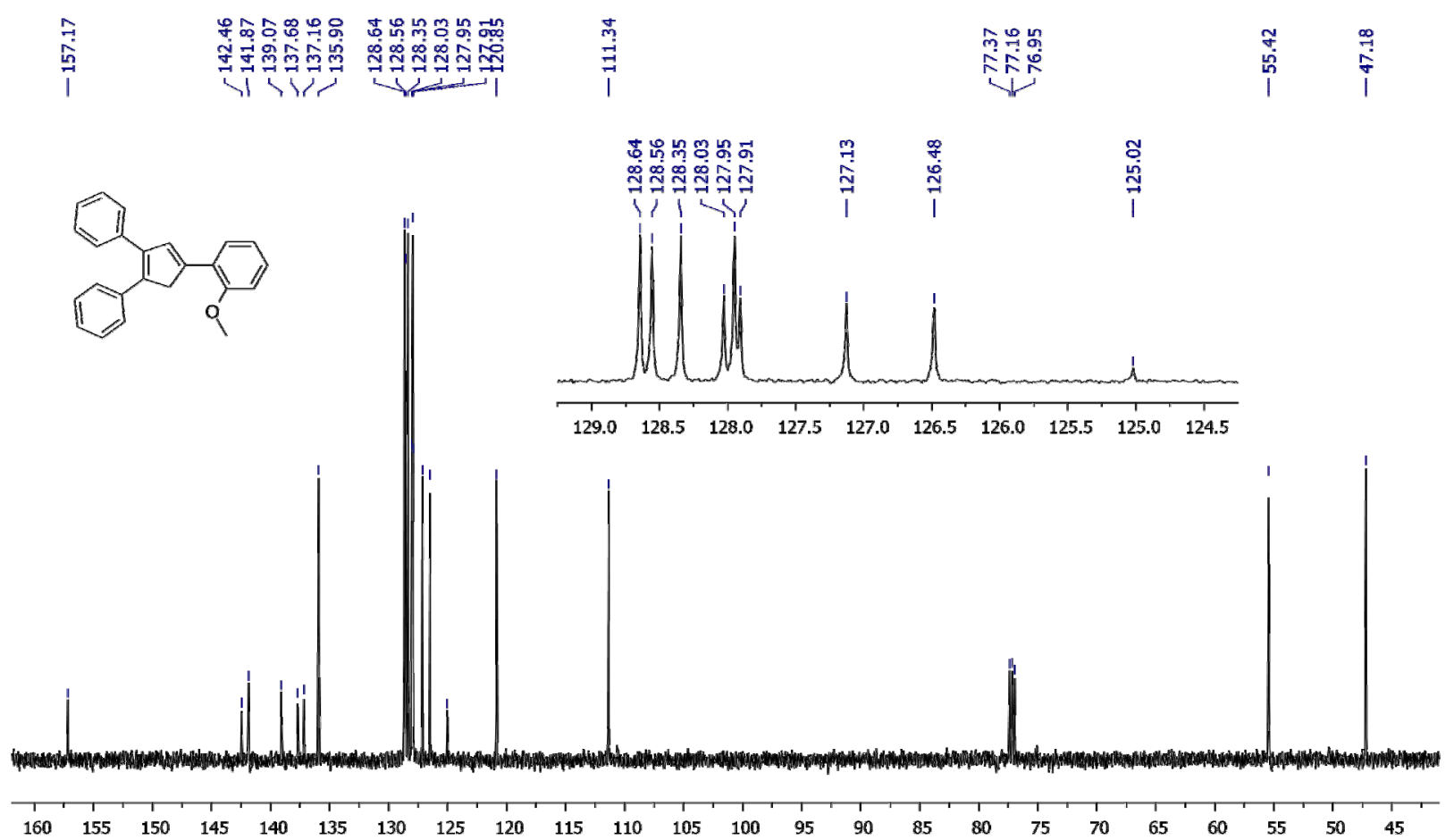

Fig. S6. ${ }^{13} \mathrm{C}\left\{{ }^{1} \mathrm{H}\right\}$ NMR spectrum $\left(\mathrm{CDCl}_{3}, 126 \mathrm{MHz}, 20^{\circ} \mathrm{C}\right)$ of $1,2-\mathrm{Ph}_{2}-4-\left(2-\mathrm{MeOC}_{6} \mathrm{H}_{4}\right) \mathrm{C}_{5} \mathrm{H}_{3}$ 


\section{S2.2. Synthesis of complexes}

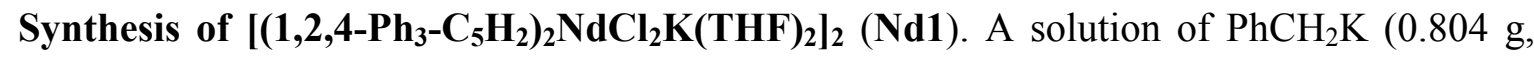
$6.17 \mathrm{mmol})$ in $30 \mathrm{~mL}$ of THF was slowly added to a $50 \mathrm{~mL}$ THF solution of $1,2,4-\mathrm{Ph}_{3} \mathrm{C}_{5} \mathrm{H}_{3}(1.785 \mathrm{~g}$, $6.06 \mathrm{mmol})$. The reaction mixture was stirred for $20 \mathrm{~min}$. The obtained solution of $\mathrm{K}\left[1,2,4-\mathrm{Ph}_{3} \mathrm{C}_{5} \mathrm{H}_{2}\right]$ was added dropwise to a stirred suspension of $\mathrm{NdCl}_{3}(\mathrm{THF})_{2.5}(1.272 \mathrm{~g}, 2.95 \mathrm{mmol})$ in THF $(20 \mathrm{~mL})$. Within a few minutes, all solids dissolved, and then precipitate started to form. The reaction mixture was stirred for $5 \mathrm{~h}$ and was then centrifuged $(5000 \mathrm{rpm}, 15 \mathrm{~min})$. The resulting solution was concentrated under vacuum to a volume of approximately 5-7 $\mathrm{mL}$. Hexane $(35 \mathrm{~mL})$ was then layered on top. Bluish crystals were obtained within 1 week. The products were washed with hexane $(3 \times 5 \mathrm{~mL})$ and dried under dynamic vacuum. The yield was $2.47 \mathrm{~g}(1.25 \mathrm{mmol}, 85 \%)$. Calcd. for $\mathrm{C}_{108} \mathrm{H}_{100} \mathrm{Cl}_{4} \mathrm{~K}_{2} \mathrm{Nd}_{2} \mathrm{O}_{4}, \%$ : C, 65.83, H, 5.12; Nd, 14.64. Found: C, 65.78; H, 5.17; Nd, 14.35 .

Synthesis of $\left\{\left[\eta^{5}-1,2-\mathrm{Ph}_{2}-4-\left(4-\mathrm{MeOC}_{6} \mathrm{H}_{4}\right) \mathrm{C}_{5} \mathrm{H}_{2}\right]_{2} \mathrm{NdCl}_{2} \mathrm{~K}(\mathrm{THF})_{2}\right\}_{2}$ (Nd2). Complex Nd2 was synthesized as described above for Nd1 from $\mathrm{PhCH}_{2} \mathrm{~K}(0.468 \mathrm{~g}, 3.59 \mathrm{mmol}), 1,2-\mathrm{Ph}_{2}-4-(4-$ $\left.\mathrm{MeOC}_{6} \mathrm{H}_{4}\right) \mathrm{C}_{5} \mathrm{H}_{3}(1.110 \mathrm{~g}, 3.42 \mathrm{mmol})$ and $\mathrm{NdCl}_{3}(\mathrm{THF})_{2.5}(0.725 \mathrm{~g}, 1.68 \mathrm{mmol})$. The yield of bluish crystals was $1.443 \mathrm{~g}(0.69 \mathrm{mmol}, 82 \%)$. Calcd. for $\mathrm{C}_{112} \mathrm{H}_{108} \mathrm{Cl}_{4} \mathrm{~K}_{2} \mathrm{Nd}_{2} \mathrm{O}_{8}, \%$ : C, 64.35; H, 5.21\%; Nd, 13.80; Found: C, 64.20; H, 5.24; Nd, 13.51.

Synthesis of $\left\{\left[1,2-\mathrm{Ph}_{2}-4-\left(2-\mathrm{MeOC}_{6} \mathrm{H}_{4}\right) \mathrm{C}_{5} \mathrm{H}_{2}\right]_{2} \mathrm{NdCl}_{2}\left[\mathrm{~K}(\mathrm{THF})_{4}\right]\right\}(\mathrm{THF})_{0.5}$ (Nd3). Complex Nd3 was synthesized as described above for $\mathbf{N d 1}$ from $\mathrm{PhCH}_{2} \mathrm{~K}\left(0.467 \mathrm{~g}, 3.59\right.$ mmol), 1,2- $\mathrm{Ph}_{2}-4-(4-$ $\left.\mathrm{MeOC}_{6} \mathrm{H}_{4}\right) \mathrm{C}_{5} \mathrm{H}_{3}(1.111 \mathrm{~g}, 3.42 \mathrm{mmol})$ and $\mathrm{NdCl}_{3}(\mathrm{THF})_{2.5}(0.724 \mathrm{~g}, 1.68 \mathrm{mmol})$. The yield of bluish crystals was $1.632 \mathrm{~g}(1.33 \mathrm{mmol}, 79 \%)$. Calcd. for $\mathrm{C}_{66} \mathrm{H}_{74} \mathrm{Cl}_{2} \mathrm{KNdO}_{6.5}, \%$ : C, 64.68; $\mathrm{H}, 6.09$; $\mathrm{Nd}$, 11.77. Found: C, 64.84; H, 5.98; Nd, 11.65 .

Synthesis of [1,2-Ph $\left.-\mathrm{Ph}_{2}-\left(\mathbf{2}-\mathrm{MeOC}_{6} \mathrm{H}_{4}\right) \mathrm{C}_{5} \mathrm{H}_{2}\right]_{2} \mathbf{N d C l}(\mathbf{N d 4})$. Complex Nd3 (306 mg, 0.25 mmol) was dissolved in toluene containing two drops of THF. The mixture was stirred for $2 \mathrm{~h}$ and then centrifuged (5000 rpm, $15 \mathrm{~min}$ ). The solvent was evaporated to $\sim 6 \mathrm{~mL}$ of residual volume. Hexane $(\sim 30 \mathrm{~mL})$ was carefully layered on top of the resulting solution. Violet octahedral crystals were formed in 6 days. Some crystals were taken for X-ray diffraction studies. The remaining crystals were separated from the mother liquor, washed with hexane and dried under vacuum. The yield of Nd4 was $184 \mathrm{mg}(0.22 \mathrm{mmol}, 89 \%)$. Calcd. for $\mathrm{C}_{48} \mathrm{H}_{38} \mathrm{ClNdO}_{2}, \%$ : C, 69.75; H, 4.63; $\mathrm{Nd}$, 17.45. Found: C, 69.85; H, 4.69; Nd, 17.54. 


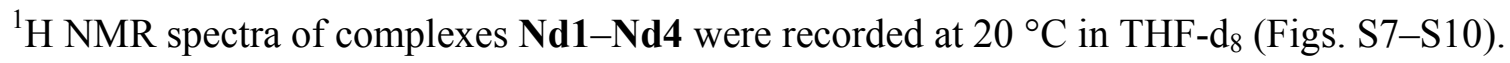
All complexes are paramagnetic. This makes it difficult to assign signals in NMR spectra.

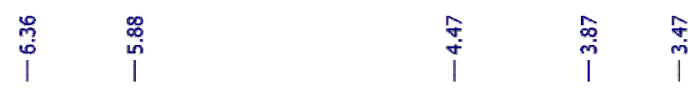

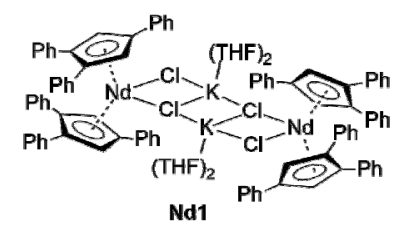

Nd1

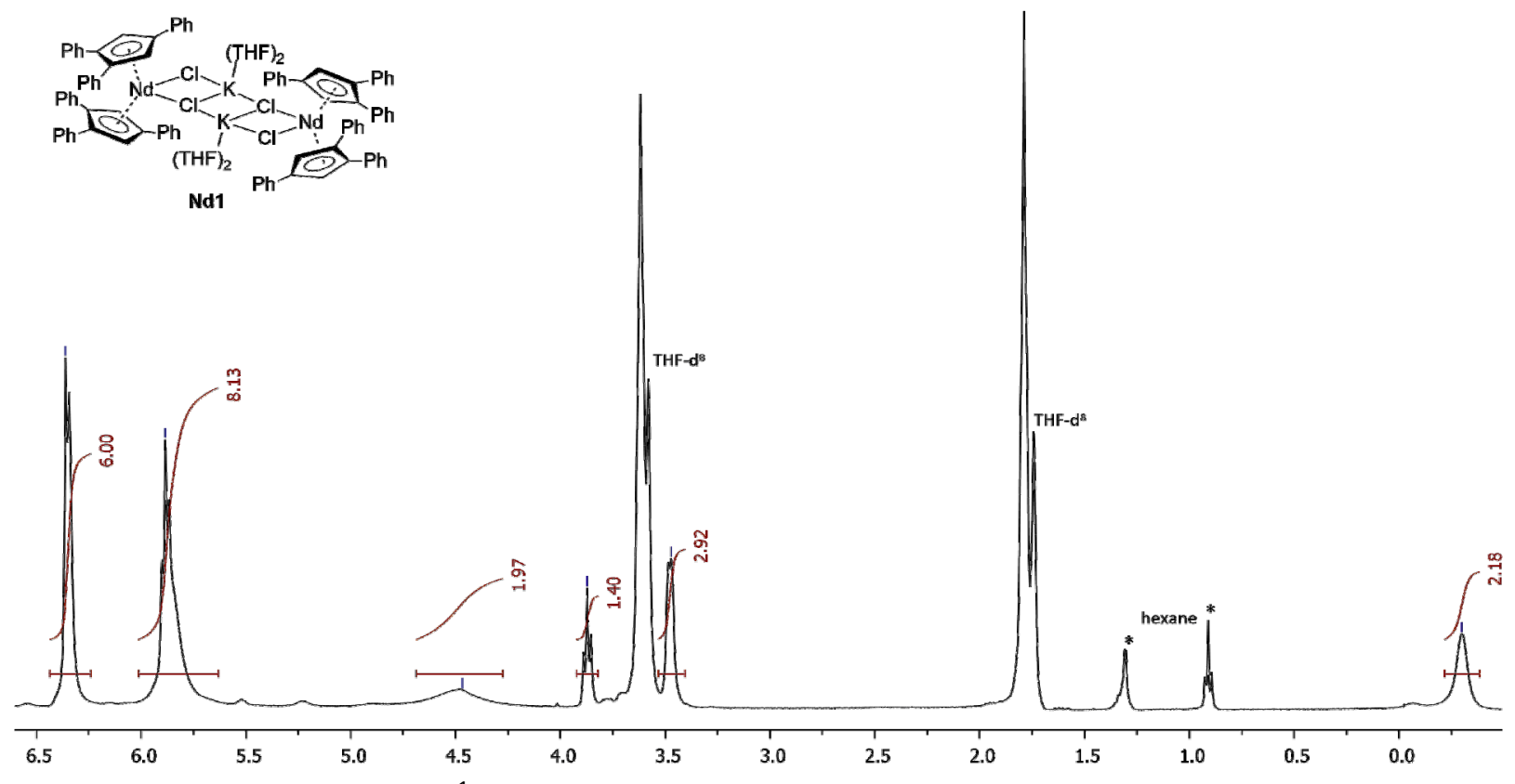

Fig. S7. ${ }^{1} \mathrm{H}$ NMR spectrum $\left(\mathrm{THF}-\mathrm{d}_{8}, 400 \mathrm{MHz}, 20^{\circ} \mathrm{C}\right)$ of $\mathbf{N d 1}$

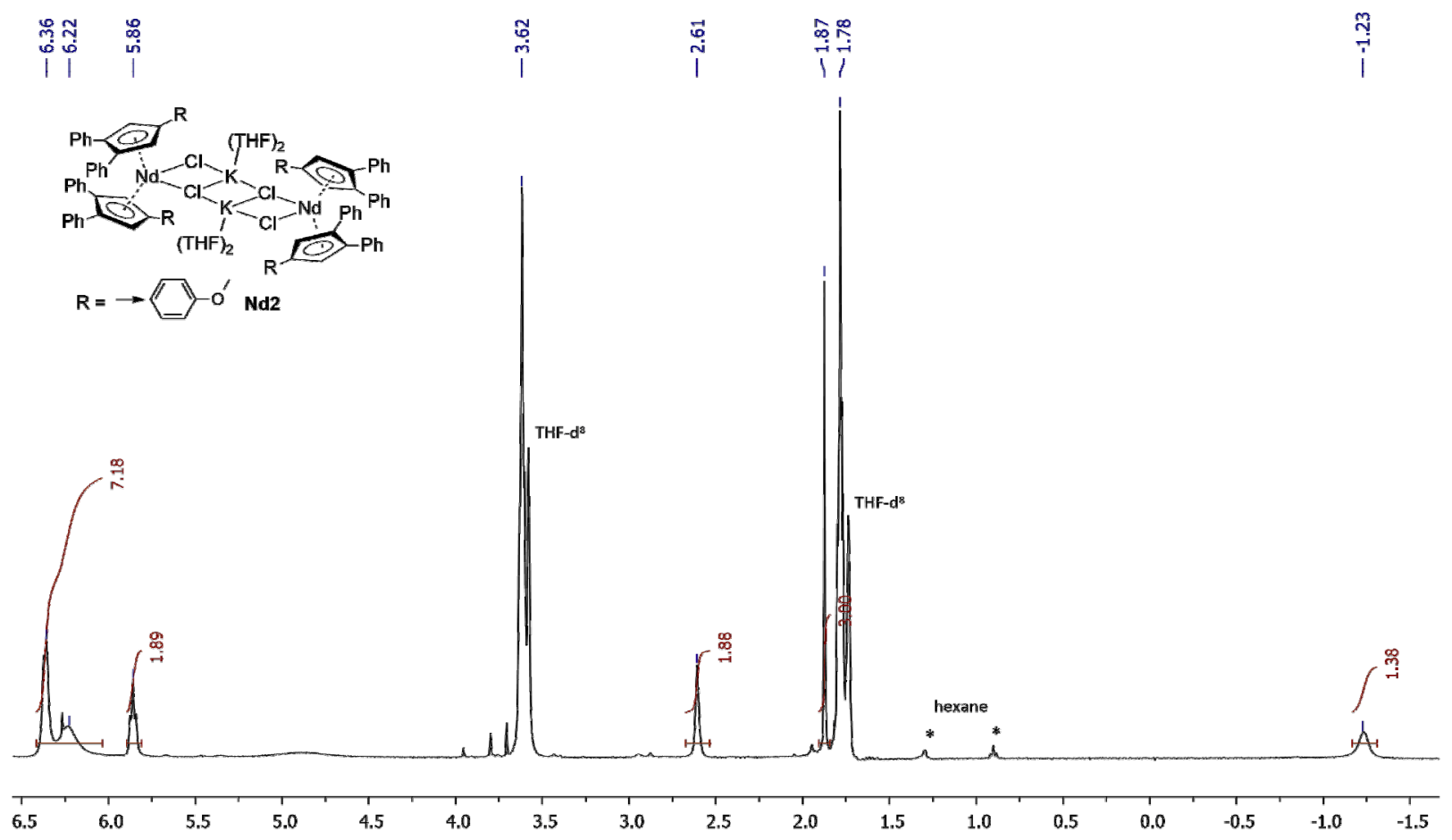

Fig. S8. ${ }^{1} \mathrm{H}$ NMR spectrum $\left(\mathrm{THF}-\mathrm{d}_{8}, 400 \mathrm{MHz}, 20^{\circ} \mathrm{C}\right)$ of $\mathbf{~ N d 2}$ 


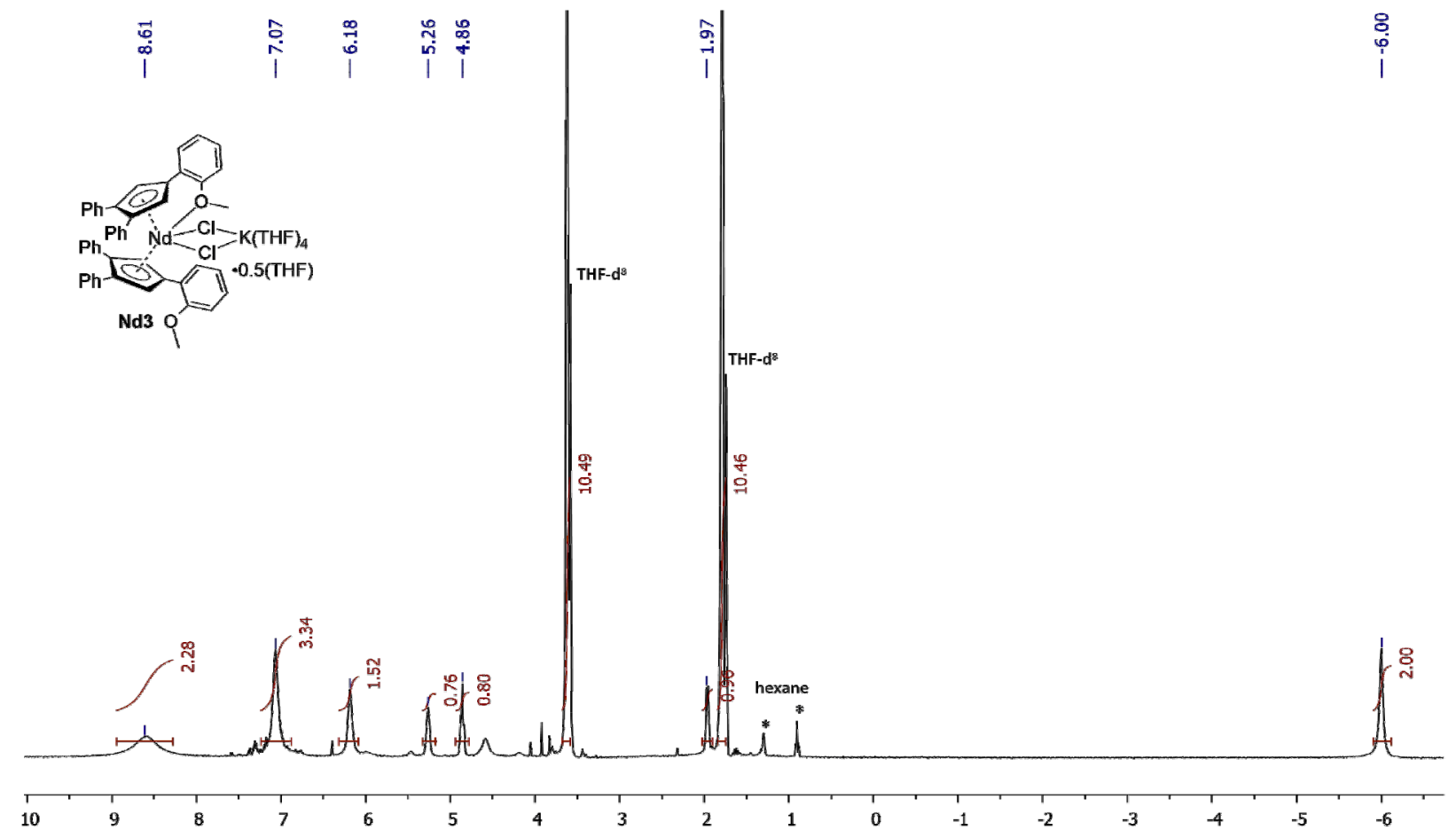

Fig. S9. ${ }^{1} \mathrm{H}$ NMR spectrum $\left(\mathrm{THF}-\mathrm{d}_{8}, 400 \mathrm{MHz}, 20^{\circ} \mathrm{C}\right)$ of $\mathbf{N d 3}$

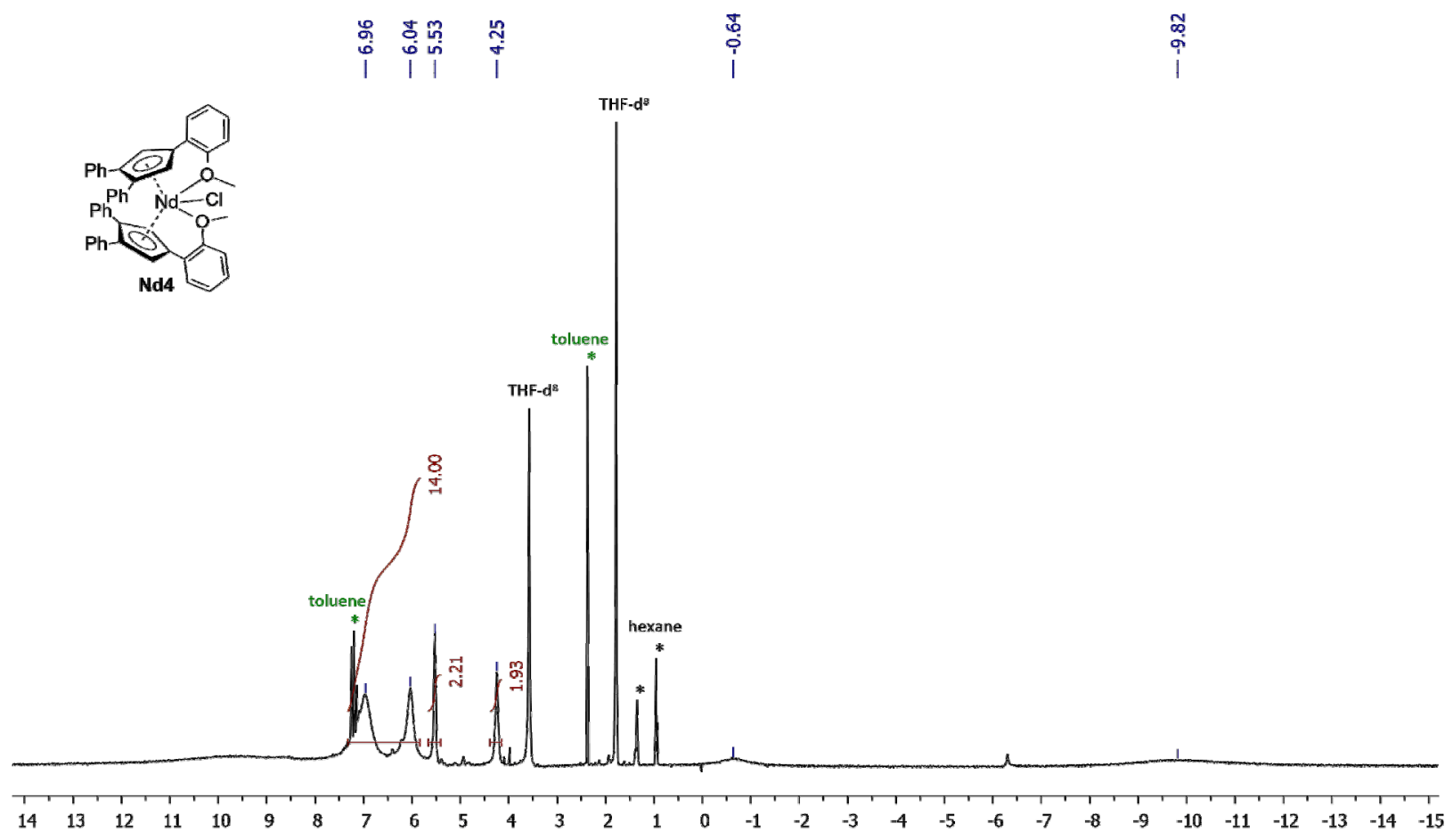

Fig. S10. ${ }^{1} \mathrm{H}$ NMR spectrum $\left(\mathrm{THF}-\mathrm{d}_{8}, 400 \mathrm{MHz}, 20^{\circ} \mathrm{C}\right.$ ) of $\mathbf{~ N d} 4$ 


\section{S3. X-ray structure determination}

\section{S3.1. X-ray diffraction experimental and refinement details, crystallographic data}

X-ray diffraction data for all studied complexes were collected using a SMART APEX II area-detector diffractometer (graphite monochromator, $\omega$-scan technique) at the temperature of 120(2) K, using MoKa $_{\mathrm{K}}$ radiation $(0.71073 \AA$ ). The intensity data were integrated by the SAINT program and corrected for absorption and decay by the multi-scan method (semi-empirical from equivalents) implemented in SADABS. ${ }^{10}$ All structures were solved by direct methods using SHELXS ${ }^{11}$ and were refined against $\mathrm{F}^{2}$ using SHELXL-2017. ${ }^{12}$ All non-hydrogen atoms were refined with anisotropic displacement parameters. All hydrogen atoms were placed in ideal calculated positions $(\mathrm{C}-\mathrm{H}$ distance $=0.980 \AA$ for methyl, $0.990 \AA$ for methylene, $0.950 \AA$ for aromatic, and $1.000 \AA$ for cyclopentadienyl hydrogen atoms) and refined as riding atoms with relative isotropic displacement parameters taken as $U_{\text {iso }}(\mathrm{H})=1.5 U_{\text {eq }}(\mathrm{C})$ for methyl $\mathrm{H}$ atoms and $U_{\text {iso }}(\mathrm{H})=1.2 U_{\text {eq }}(\mathrm{C})$ otherwise. The studied crystal of $\mathbf{N d 4}$ was refined as a racemic twin with the ratio for two components being of 0.984(16):0.016(16). The SHELXTL program suite ${ }^{13}$ was used for molecular graphics. Crystal data, data collection and structure refinement details are summarized in Table S1. The description of the structures Nd1-Nd4, ORTEP drawings, and selected bond distances can be found below in Sections S3.2-S3.5. 
Table S1. X-ray crystallographic data and refinement details for studied complexes

\begin{tabular}{|c|c|c|c|c|}
\hline & Nd1 & Nd2 & Nd3 & Nd4 \\
\hline Empirical formula & $\mathrm{C}_{108} \mathrm{H}_{1000} \mathrm{Cl}_{4} \mathrm{~K}_{2} \mathrm{Nd}_{2} \mathrm{O}_{4}$ & $\mathrm{C}_{112} \mathrm{H}_{108} \mathrm{Cl}_{4} \mathrm{~K}_{2} \mathrm{Nd}_{2} \mathrm{O}_{8}$ & $\mathrm{C}_{66} \mathrm{H}_{74} \mathrm{Cl}_{2} \mathrm{KNdO}_{6.50}$ & $\mathrm{C}_{48} \mathrm{H}_{38} \mathrm{CINdO}$ \\
\hline Formula weight & 1970.35 & 2090.46 & 1225.49 & 826.47 \\
\hline Crystal system & Triclinic & Monoclinic & Triclinic & Tetragonal \\
\hline Space group & $\mathrm{P} \overline{1}$ & $\mathrm{P} 2_{1} / \mathrm{c}$ & $\mathrm{P} \overline{1}$ & $\mathrm{P} 4_{1} 2_{1} 2$ \\
\hline \multicolumn{5}{|l|}{ Unit cell dimensions } \\
\hline $\mathrm{a}(\mathrm{A})$ & $13.1068(6)$ & $14.6632(6)$ & $12.393(7)$ & $9.9104(4)$ \\
\hline$b(\AA ̊)$ & $13.5864(7)$ & $13.2422(5)$ & $12.639(7)$ & $9.9104(4)$ \\
\hline$c(\AA ̊)$ & $14.2130(6)$ & $25.1879(10)$ & $19.542(10)$ & $39.1176(16)$ \\
\hline 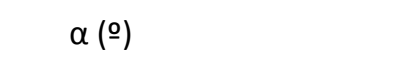 & 63.6492(9) & 90 & $89.432(9)$ & 90 \\
\hline 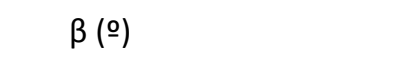 & $89.3990(10)$ & $96.0960(10)$ & $80.143(10)$ & 90 \\
\hline  & $89.5451(11)$ & 90 & $80.218(12)$ & 90 \\
\hline Volume $\left(\AA^{3}\right)$ & $2267.84(18)$ & $4863.2(3)$ & 2971(3) & $3842.0(3)$ \\
\hline z & 1 & 2 & 2 & 4 \\
\hline$D_{\text {calcd }}\left(g / \mathrm{cm}^{3}\right)$ & 1.443 & 1.428 & 1.370 & 1.429 \\
\hline$\mu\left(\mathrm{mm}^{-1}\right)$ & 1.395 & 1.309 & 1.085 & 1.460 \\
\hline$F(000)$ & 1006 & 2140 & 1270 & 1676 \\
\hline$\Theta$ Range (o) & 2.22 to 30.00 & 1.40 to 30.00 & 1.69 to 30.00 & 2.08 to 29.00 \\
\hline Complentess to $\Theta_{\max }$ & 0.997 & 1.000 & 1.000 & 1.000 \\
\hline \multirow[t]{3}{*}{ Index ranges } & $-18 \leq h \leq 12$ & $-20 \leq h \leq 20$ & $-17 \leq h \leq 17$ & $-13 \leq h \leq 13$ \\
\hline & $-19 \leq k \leq 19$ & $-18 \leq k \leq 18$ & $-17 \leq k \leq 17$ & $-13 \leq k \leq 13$ \\
\hline & $-19 \leq 1 \leq 19$ & $-35 \leq 1 \leq 35$ & $-27 \leq 1 \leq 27$ & $-53 \leq 1 \leq 53$ \\
\hline Reflections collected & 22320 & 62200 & 40111 & 77516 \\
\hline Reflections unique $\left(R_{\text {int }}\right)$ & $13047(0.0340)$ & $14194(0.0297)$ & $17331(0.0312)$ & $5125(0.0369)$ \\
\hline Reflections with $\mid>2 \sigma(I)$ & 10679 & 12574 & 14603 & 5082 \\
\hline Variables / restraints & $566 / 19$ & $592 / 0$ & $759 / 79$ & $238 / 0$ \\
\hline Flack & - & - & - & $0.016(16)$ \\
\hline Goodness-of-fit on $F^{2}$ & 1.003 & 1.023 & 1.024 & 1.050 \\
\hline $\begin{array}{l}\text { Final } R_{1}, w R_{2} \text { indices with } \\
\mid>2 \sigma(I)\end{array}$ & $0.0383,0.0761$ & $0.0239,0.0538$ & $0.0398,0.0940$ & $0.0237,0.0655$ \\
\hline $\mathrm{R}_{1}, \mathrm{wR}_{2}$ indices (all data) & $0.0536,0.0818$ & $0.0295,0.0564$ & $0.0512,0.1006$ & $0.0240,0.0657$ \\
\hline $\begin{array}{l}\text { Largest difference in peak / } \\
\text { hole }\left(e / \AA^{3}\right)\end{array}$ & $0.902 /-0.627$ & $0.681 /-0.488$ & $1.020 /-0.598$ & $0.888 /-1.226$ \\
\hline CCDC number & 1870072 & 1870073 & 1870074 & 1870075 \\
\hline
\end{tabular}




\section{S3.2. $\left[\left(\eta^{5}-1,2,4-\mathrm{Ph}_{3}-\mathrm{C}_{5} \mathrm{H}_{2}\right)_{2} \mathrm{NdCl}_{2} \mathrm{~K}(\mathrm{THF})_{2}\right]_{2}, \mathrm{Nd} 1$}

Complex $\left[\left(\eta^{5}-1,2,4-\mathrm{Ph}_{3}-\mathrm{C}_{5} \mathrm{H}_{2}\right)_{2} \mathrm{Nd}\left(\mu_{2}-\mathrm{Cl}\right)\left(\mu_{3}-\mathrm{Cl}\right) \mathrm{K}(\mathrm{THF})_{2}\right]_{2}$ (Fig. S11) is located at an inversion center. Half a molecule is crystallographically unique. No non-coordinating solvent molecule is present. $\mathrm{Nd}^{3+}\left(\mathrm{CN}_{\mathrm{Nd}}=8\right)$ is coordinated by two $\eta^{5}$ cyclopentadienyl ligands and by two $\mathrm{Cl}^{-}$anions. The $\mathrm{Cp}_{\text {(centroid) }} \mathrm{Nd}-\mathrm{Cp}$ (centroid) angle is $128.4^{\circ}$. The dihedral angle between two $\mathrm{Cp}$ planes is $53.01(12)^{\circ}$. The $\mathrm{K}^{+}$cation $\left(\mathrm{CN}_{\mathrm{K}}=5\right)$ is coordinated by three $\mathrm{Cl}^{-}$ligands and two THF molecules, which are disordered over two positions with corresponding ratios of 0.73(1):0.27(1) for atoms C47-C50 and 0.762(7):0.238(7) for C51-C53. Being coordinatively unsaturated, $\mathrm{K}^{+}$ additionally has long contacts to atoms $\mathrm{C} 37$ and $\mathrm{C} 38$ of the $\pi$-system of one $\mathrm{Ph}$ group. The complex has a $\mathrm{Nd}_{2} \mathrm{Cl}_{4} \mathrm{~K}_{2}$ core (Fig. S12). The same type of the core was previously described for similar rareearth complexes. ${ }^{6,14}$ The $\mathrm{K}_{2} \mathrm{Cl}_{2}$ and $\mathrm{KCl}_{2} \mathrm{Nd}$ fragments are planar with the dihedral angle of $38.61(3)^{\circ}$.

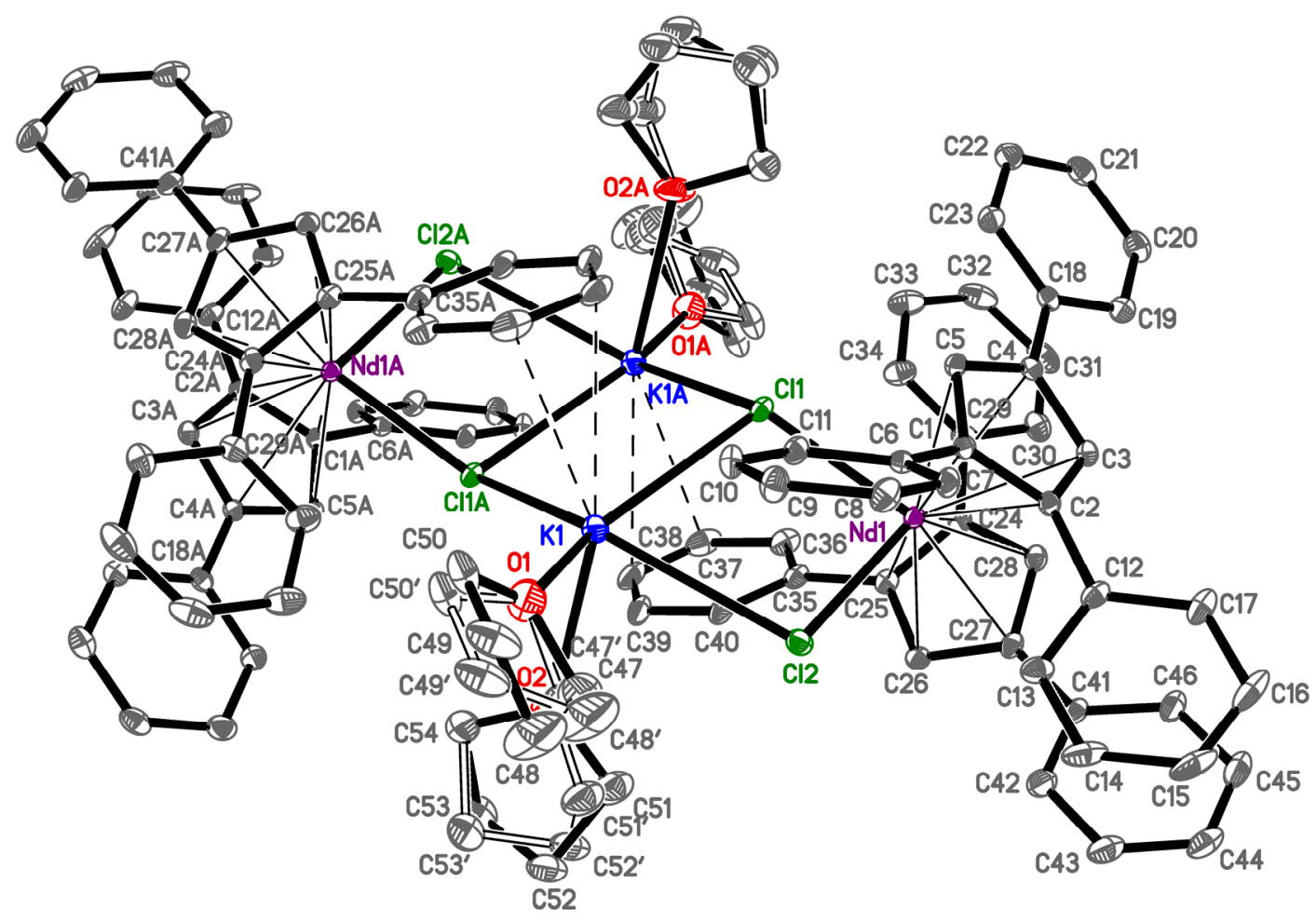

Figure S11. The structure of Nd1. Thermal ellipsoids are set to the $30 \%$ probability level, $\mathrm{H}$ atoms are omitted for clarity. Disordered fragments are shown with open solid lines. Symmetry code: A $-x+1,-y+1,-z+2$ 


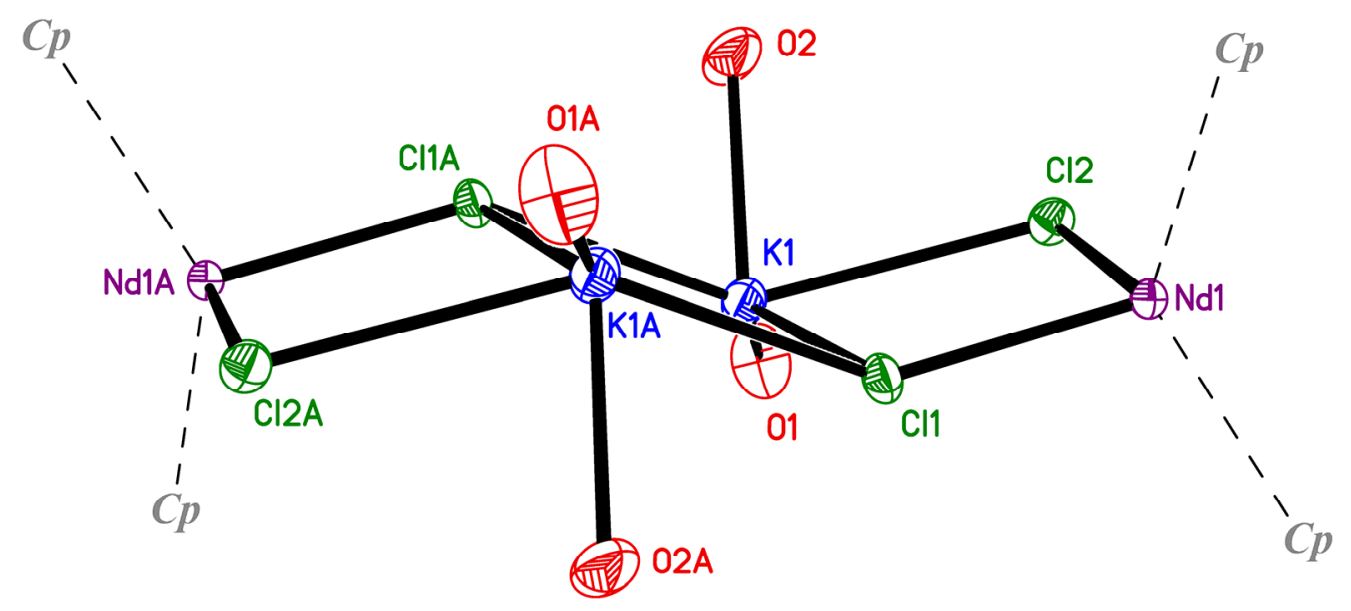

Figure S12. The core structure in Nd1. Thermal ellipsoids are set to the $50 \%$ probability level

Table S2. Selected distances $(\AA)$ and angles $\left(^{\circ}\right)$ in Nd1

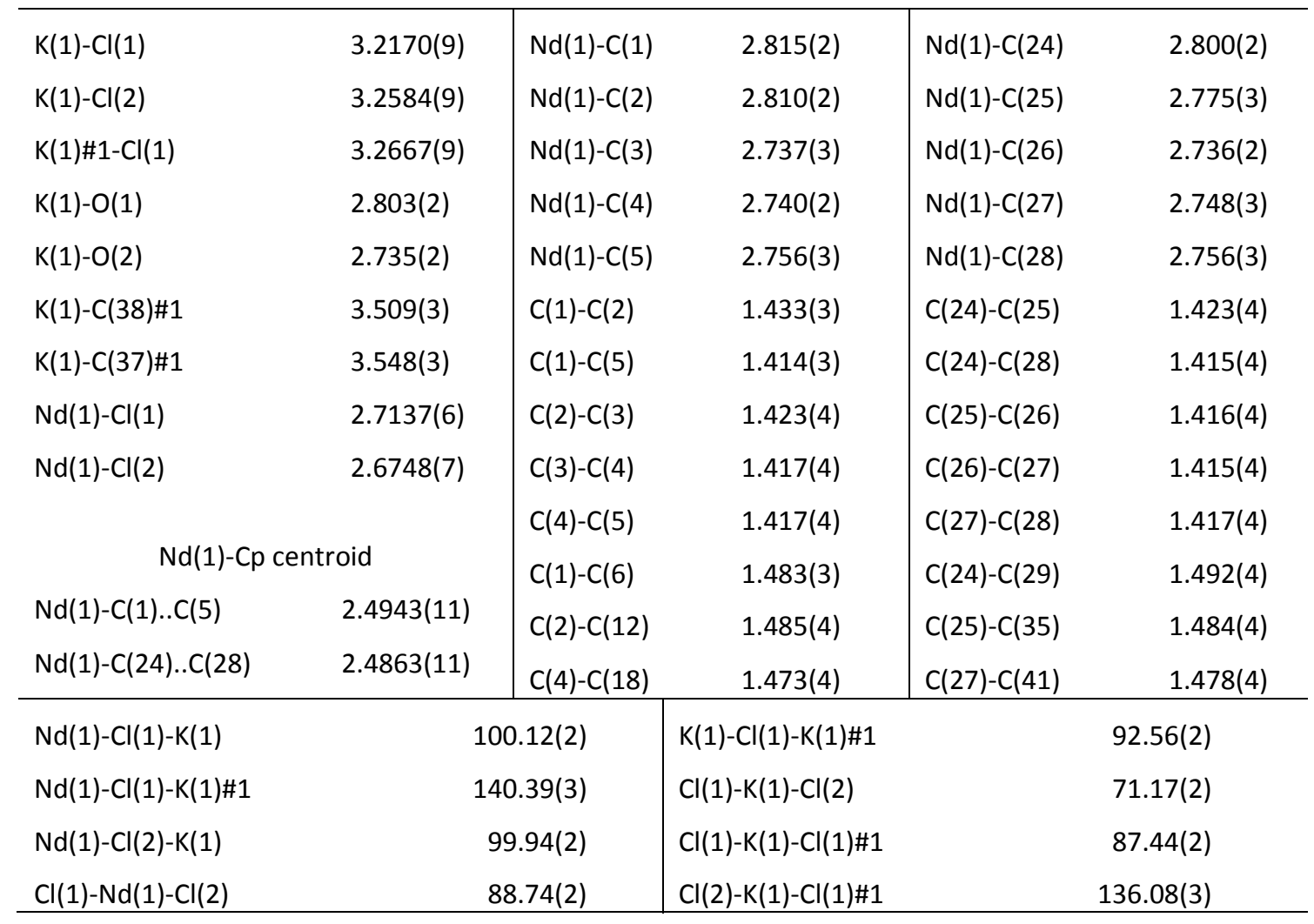

Symmetry transformation to generate equivalent atoms: $\# 1-\mathrm{x}+1,-\mathrm{y}+1,-\mathrm{z}+2$ (an inversion center) 
Table S3. Dihedral angles between the $\mathrm{Cp}$ and $\mathrm{Ph}$ planes $\left({ }^{\circ}\right)$ and deviations of $\mathrm{C}_{\mathrm{ipso}}(\mathrm{Ph})$ atoms from the corresponding $\mathrm{Cp}$ plane $(\AA)$ in Nd1

\begin{tabular}{lllll}
\hline $\mathrm{Cp}$ & $\mathrm{Ph}$ & $\mathrm{Cp}-\mathrm{Ph}$ & $\mathrm{C}_{\mathrm{ipso}}(\mathrm{Ph})$ & Deviation \\
\hline $\mathrm{C}(1) . . \mathrm{C}(5)$ & $\mathrm{C}(6) . . \mathrm{C}(11)$ & $47.31(10)$ & $\mathrm{C}(6)$ & $0.166(4)$ \\
$\mathrm{C}(1) . . \mathrm{C}(5)$ & $\mathrm{C}(12) . . \mathrm{C}(17)$ & $34.76(12)$ & $\mathrm{C}(12)$ & $0.117(4)$ \\
$\mathrm{C}(1) . . \mathrm{C}(5)$ & $\mathrm{C}(18) . . \mathrm{C}(23)$ & $3.32(10)$ & $\mathrm{C}(18)$ & $0.064(5)$ \\
$\mathrm{C}(24) . . \mathrm{C}(28)$ & $\mathrm{C}(29) . . \mathrm{C}(34)$ & $44.57(12)$ & $\mathrm{C}(29)$. & $0.126(5)$ \\
$\mathrm{C}(24) . . \mathrm{C}(28)$ & $\mathrm{C}(35) . . \mathrm{C}(40)$ & $37.52(13)$ & $\mathrm{C}(35)$ & $0.068(5)$ \\
$\mathrm{C}(24) . . \mathrm{C}(28)$ & $\mathrm{C}(41) . . \mathrm{C}(46)$ & $22.74(15)$ & $\mathrm{C}(41)$ & $0.042(5)$ \\
\hline
\end{tabular}

\section{S3.3. $\left\{\left[\eta^{5}-1,2-\mathrm{Ph}_{2}-4-\left(4-\mathrm{MeOC}_{6} \mathrm{H}_{4}\right) \mathrm{C}_{5} \mathrm{H}_{2}\right]_{2} \mathrm{Nd}\left(\mu_{2}-\mathrm{Cl}\right)\left(\mu_{3}-\mathrm{Cl}\right) \mathrm{K}(\mathrm{THF})_{2}\right\}_{2}, \mathrm{Nd} 2$}

Complex $\left\{\left[\eta^{5}-1,2-\mathrm{Ph}_{2}-4-\left(4-\mathrm{MeOC}_{6} \mathrm{H}_{4}\right) \mathrm{C}_{5} \mathrm{H}_{2}\right]_{2} \mathrm{Nd}\left(\mu_{2}-\mathrm{Cl}\right)\left(\mu_{3}-\mathrm{Cl}\right) \mathrm{K}(\mathrm{THF})_{2}\right\}_{2}$ (Fig. S13) is also located at an inversion center and has a very similar structure compared with Nd1 and the same core (Fig. S14). The $\mathrm{Cp}_{\text {(centroid) }} \mathrm{Nd}-\mathrm{Cp}$ (centroid) angle is $129.9^{\circ}$. The dihedral angle between two $\mathrm{Cp}$ planes is $53.29(7)^{\circ}$. The only difference is that $\mathrm{NdCl}_{2} \mathrm{~K}$ fragment is not planar- the angle between two $\mathrm{NdClK}$ fragments is $38.26(5)^{\circ}$. $\mathrm{K}^{+}$is disordered over two positions with the probability ratio of 0.963(11):0.037(11) for $\mathrm{K}(1)$ and $\mathrm{K}\left(1^{\prime}\right)$. Atom $\mathrm{C}(55)$ of one coordinated THF molecule is also disordered with the 0.691(11):0.309(11) ratio.

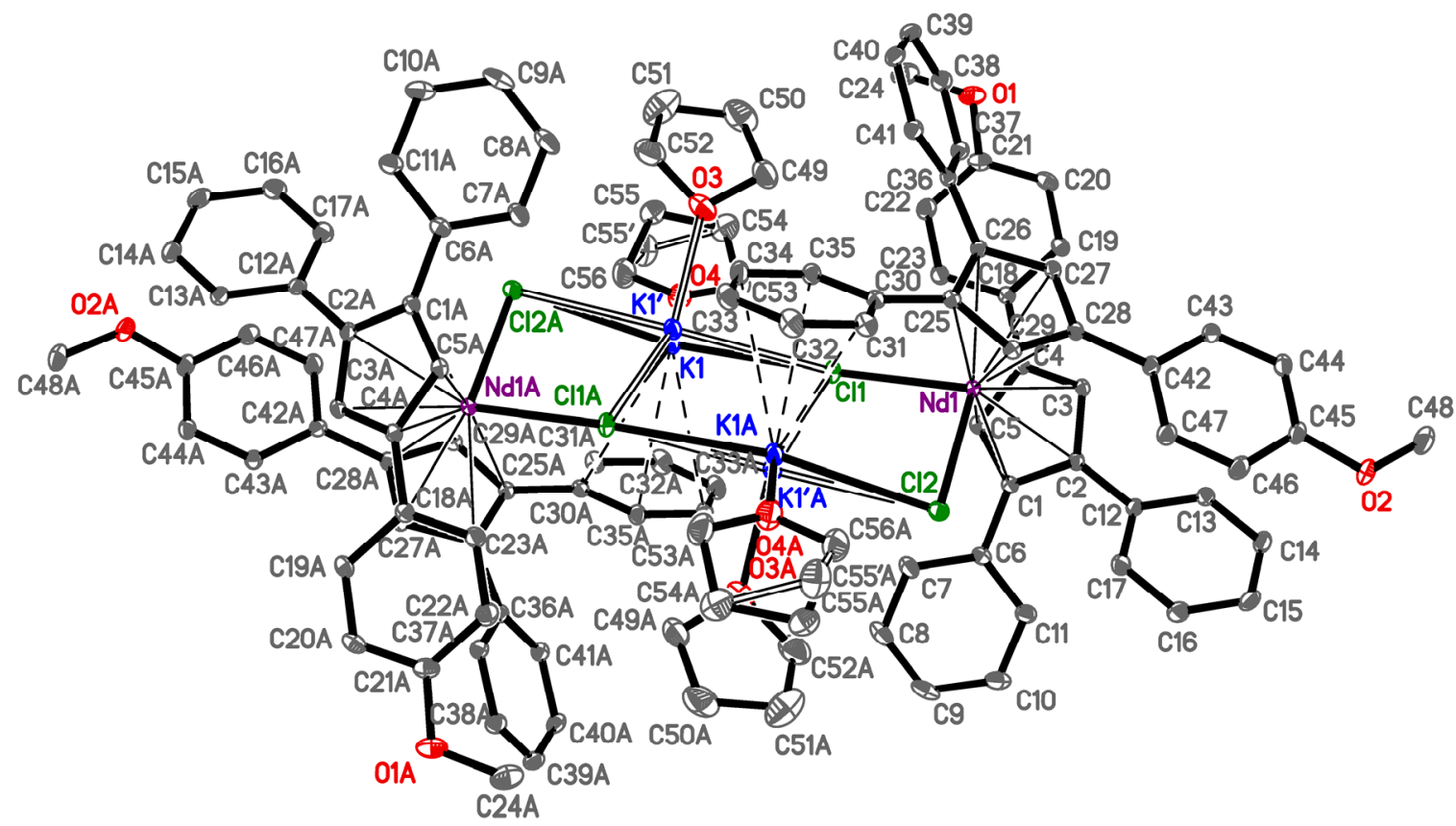

Figure S13. The structure of $\mathbf{N d 2}$ Thermal ellipsoids are set to the $30 \%$ probability level, $\mathrm{H}$ atoms are omitted for clarity. Disordered fragments are shown with open solid lines. Symmetry code: A - $x+1,-y+1,-z+1$ 


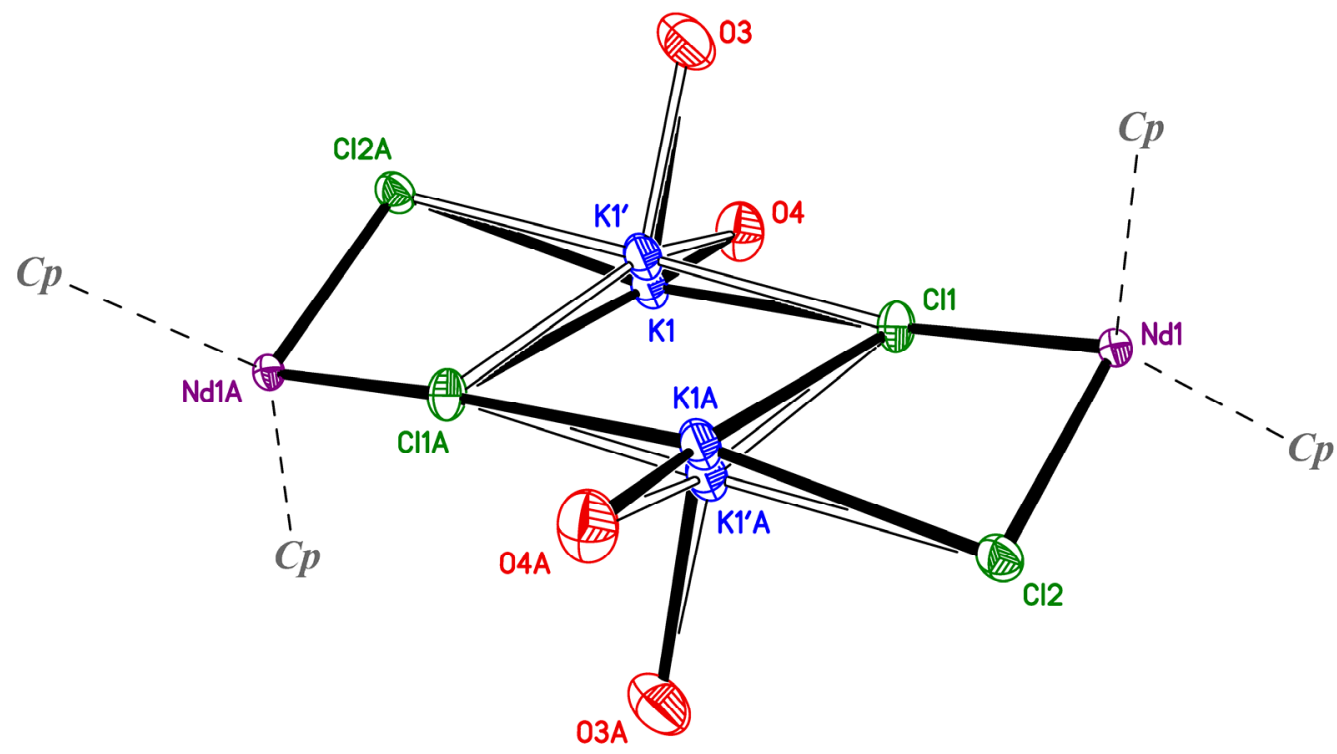

Figure S14. The core structure in Nd2. Thermal ellipsoids are set to the 50\% probability level. Disordered fragments are shown with open solid lines

Table S4. Selected distances $(\AA)$ and angles $\left(^{\circ}\right)$ in Nd2

\begin{tabular}{|c|c|c|c|c|c|}
\hline $\mathrm{K}(1)-\mathrm{Cl}(1)$ & $3.1162(7)$ & $\mathrm{Nd}(1)-\mathrm{C}(1)$ & $2.8205(15)$ & $\mathrm{Nd}(1)-\mathrm{C}(25)$ & $2.8150(15)$ \\
\hline $\mathrm{K}(1) \# 1-\mathrm{Cl}(1)$ & $3.2316(10)$ & $\mathrm{Nd}(1)-\mathrm{C}(2)$ & $2.7942(15)$ & $\mathrm{Nd}(1)-\mathrm{C}(26)$ & $2.8484(15)$ \\
\hline $\mathrm{K}(1) \# 1-\mathrm{Cl}(2)$ & $3.1436(9)$ & $\mathrm{Nd}(1)-\mathrm{C}(3)$ & $2.7209(15)$ & $\mathrm{Nd}(1)-\mathrm{C}(27)$ & $2.7677(15)$ \\
\hline $\mathrm{K}(1)-\mathrm{O}(3)$ & $2.718(3)$ & $\mathrm{Nd}(1)-\mathrm{C}(4)$ & $2.7204(15)$ & $\mathrm{Nd}(1)-\mathrm{C}(28)$ & $2.7420(15)$ \\
\hline $\mathrm{K}(1)-\mathrm{O}(4)$ & $2.616(2)$ & $\mathrm{Nd}(1)-\mathrm{C}(5)$ & $2.7611(15)$ & $\mathrm{Nd}(1)-\mathrm{C}(29)$ & $2.7471(15)$ \\
\hline $\mathrm{K}(1)-C(35) \# 1$ & $3.293(3)$ & $C(1)-C(2)$ & $1.432(2)$ & $C(25)-C(26)$ & $1.431(2)$ \\
\hline $\mathrm{K}(1)-\mathrm{C}(34) \# 1$ & $3.390(3)$ & $C(1)-C(5)$ & $1.419(2)$ & $C(25)-C(29)$ & $1.420(2)$ \\
\hline$K(1)-C(30) \# 1$ & $3.419(3)$ & $C(2)-C(3)$ & $1.420(2)$ & $C(26)-C(27)$ & $1.419(2)$ \\
\hline $\mathrm{Nd}(1)-\mathrm{Cl}(1)$ & 2.7099(4) & $C(3)-C(4)$ & $1.425(2)$ & $C(27)-C(28)$ & $1.418(2)$ \\
\hline $\mathrm{Nd}(1)-\mathrm{Cl}(2)$ & $2.6789(4)$ & $C(4)-C(5)$ & $1.418(2)$ & $C(28)-C(29)$ & $1.420(2)$ \\
\hline \multicolumn{2}{|c|}{$\mathrm{Nd}(1)-\mathrm{Cp}$ centroid } & $C(1)-C(6)$ & $1.480(2)$ & $C(25)-C(30)$ & $1.480(2)$ \\
\hline $\mathrm{Nd}(1)-\mathrm{C}(1) . . \mathrm{C}(5)$ & $2.4845(7)$ & $C(2)-C(12)$ & $1.482(2)$ & $C(26)-C(36)$ & $1.479(2)$ \\
\hline $\mathrm{Nd}(1)-\mathrm{C}(25) . . \mathrm{C}(29)$ & $2.5081(7)$ & $C(4)-C(18)$ & $1.475(2)$ & $C(28)-C(42)$ & $1.478(2)$ \\
\hline $\mathrm{Nd}(1)-\mathrm{Cl}(1)-\mathrm{K}(1)$ & \multicolumn{2}{|c|}{$171.40(5)$} & \multicolumn{2}{|l|}{$\mathrm{K}(1)-\mathrm{Cl}(1)-\mathrm{K}(1) \# 1$} & $83.70(3)$ \\
\hline $\mathrm{Nd}(1)-\mathrm{Cl}(1)-\mathrm{K}(1) \# 1$ & \multicolumn{2}{|c|}{$90.607(19)$} & \multicolumn{2}{|l|}{$\mathrm{Cl}(1)-\mathrm{K}(1)-\mathrm{Cl}(2) \# 1$} & $160.14(10)$ \\
\hline $\mathrm{Nd}(1)-\mathrm{Cl}(2)-\mathrm{K}(1) \# 1$ & \multicolumn{2}{|c|}{$93.111(18)$} & \multicolumn{2}{|l|}{$\mathrm{Cl}(1)-\mathrm{K}(1)-\mathrm{Cl}(1) \# 1$} & $96.30(3)$ \\
\hline $\mathrm{Cl}(2)-\mathrm{Nd}(1)-\mathrm{Cl}(1)$ & \multicolumn{2}{|c|}{$90.463(13)$} & \multicolumn{2}{|c|}{$\mathrm{Cl}(2) \# 1-\mathrm{K}(1)-\mathrm{Cl}(1) \# 1$} & $73.74(2)$ \\
\hline
\end{tabular}

Symmetry transformation to generate equivalent atoms: $\# 1-\mathrm{x}+1,-\mathrm{y}+1,-\mathrm{z}+1$ (an inversion center) 
Table S5. Dihedral angles between the $\mathrm{Cp}$ and $\mathrm{Ph}$ planes $\left(^{\circ}\right)$ and deviations of $\mathrm{C}_{\mathrm{ipso}}(\mathrm{Ph})$ atoms from the Cp plane $(\AA)$ in $\mathbf{N d 2}$

\begin{tabular}{lllll}
\hline $\mathrm{Cp}$ & $\mathrm{Ph}$ & $\mathrm{Cp}-\mathrm{Ph}$ & $\mathrm{C}_{\mathrm{ipso}}(\mathrm{Ph})$ & Deviation \\
\hline $\mathrm{C}(1) . . \mathrm{C}(5)$ & $\mathrm{C}(6) . . \mathrm{C}(11)$ & $36.26(6)$ & $\mathrm{C}(6)$ & $0.140(3)$ \\
$\mathrm{C}(1) . . \mathrm{C}(5)$ & $\mathrm{C}(12) . . \mathrm{C}(17)$ & $43.03(7)$ & $\mathrm{C}(12)$ & $0.144(3)$ \\
$\mathrm{C}(1) . . \mathrm{C}(5)$ & $\mathrm{C}(18) . . \mathrm{C}(23)$ & $19.44(7)$ & $\mathrm{C}(18)$ & $0.022(3)$ \\
$\mathrm{C}(25) . . \mathrm{C}(29)$ & $\mathrm{C}(30) . . \mathrm{C}(35)$ & $29.93(7)$ & $\mathrm{C}(30)$ & $0.018(3)$ \\
$\mathrm{C}(25) . . \mathrm{C}(29)$ & $\mathrm{C}(36) . . \mathrm{C}(41)$ & $46.11(4)$ & $\mathrm{C}(36)$ & $0.372(3)$ \\
$\mathrm{C}(25) . . \mathrm{C}(29)$ & $\mathrm{C}(42) . . \mathrm{C}(47)$ & $10.76(9)$ & $\mathrm{C}(42)$ & $0.096(3)$ \\
\hline
\end{tabular}

\section{S3.4. $\left\{\left[\eta^{5}-1,2-\mathrm{Ph}_{2}-4-\left(2-\mathrm{MeOC}_{6} \mathrm{H}_{4}\right) \mathrm{C}_{5} \mathrm{H}_{2}\right]_{2} \mathrm{NdCl}_{2}\left[\mathrm{~K}(\mathrm{THF})_{4}\right]\right\}(\mathrm{THF})_{0.5}, \mathrm{Nd3}$}

The ate complex $\left\{\left[\eta^{5}-1,2-\mathrm{Ph}_{2}-4-\left(2-\mathrm{MeOC}_{6} \mathrm{H}_{4}\right) \mathrm{C}_{5} \mathrm{H}_{2}\right]_{2} \mathrm{Nd}(\mu-\mathrm{Cl})_{2}\left[\mathrm{~K}(\mathrm{THF})_{4}\right]\right\}(\mathrm{THF})_{0.5}$ (Fig. S15) contains a $\left[\mathrm{Nd}(\mu-\mathrm{Cl})_{2} \mathrm{~K}\right]$ core. The $\mathrm{Nd}^{3+}$ is coordinated by two $\eta^{5}$ cyclopentadienyl ligands with the $\mathrm{Cp}$ (centroid) $-\mathrm{Nd}-\mathrm{Cp}_{\text {(centroid) }}$ angle of $128.2^{\circ}$. The dihedral angle between two $\mathrm{Cp}$ planes is $56.54(11)^{\circ}$. The methoxy group of one $\mathrm{Cp}$ ligand has a long contact to the $\mathrm{Nd}^{3+}$ cation (Table $\mathrm{S} 6$ ), whereas the second methoxy group remains non-coordinated. $\mathrm{Nd}^{3+}$ is also coordinated by two $\mathrm{Cl}^{-}$anions $\left(\mathrm{CN}_{\mathrm{Nd}}=9\right)$ that form bridges to a complex $\left[\mathrm{K}(\mathrm{THF})_{4}\right]^{+}$cation $\left(\mathrm{CN}_{\mathrm{K}}=6\right)$. The $\left[\mathrm{K}(\mathrm{THF})_{4}\right]^{+}$cation is disordered over two positions. A non-coordinating THF molecule is located at an inversion center with $50 \%$ atom site occupancies.

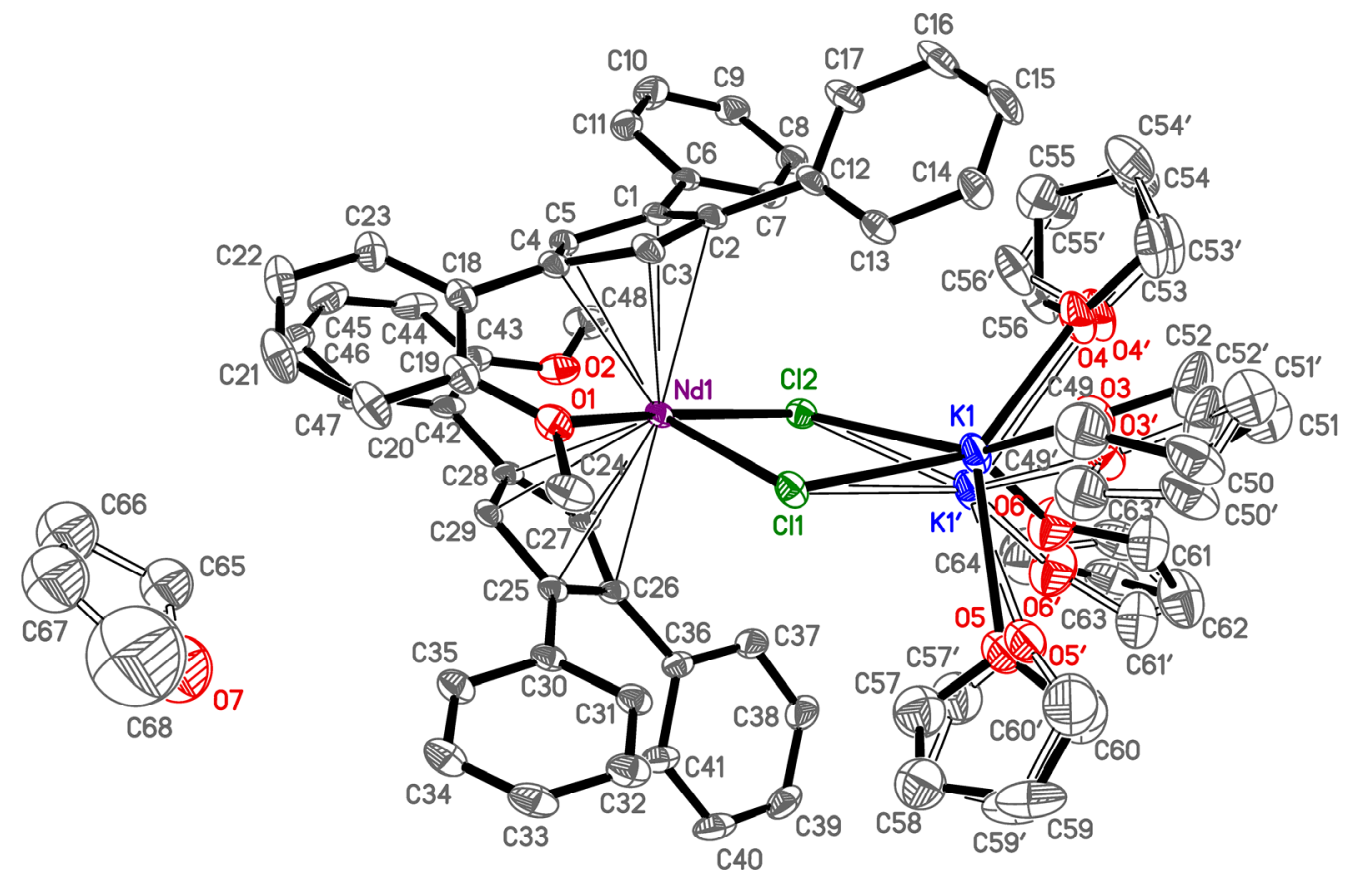

Figure S15. The structure of Nd3. Thermal ellipsoids are set to the $30 \%$ probability level, $\mathrm{H}$ atoms are omitted for clarity. Disordered fragments are shown with open solid lines 
The angles between C11-Nd1-Cl2 plane and planes formed by Cl1-K1-Cl2 and Cl1-K1'-Cl2 are $13.75(10)^{\circ}$ and $1.0(4)^{\circ}$, respectively. Some weak $\mathrm{C}-\mathrm{H}_{(\mathrm{THF}) .} . \mathrm{Cl}$ and $\mathrm{C}-\mathrm{H}_{(\mathrm{THF}) . .} \mathrm{O}_{(\mathrm{THF})}$ hydrogen bonds have been found (Table S8).

Table S6. Selected distances $(\AA)$ and angles $\left(^{\circ}\right)$ in Nd3.

\begin{tabular}{lc|ll|ll}
\hline $\mathrm{K}(1)-\mathrm{Cl}(1)$ & $3.1001(17)$ & $\mathrm{Nd}(1)-\mathrm{C}(1)$ & $2.923(3)$ & $\mathrm{Nd}(1)-\mathrm{C}(25)$ & $2.849(3)$ \\
$\mathrm{K}(1)-\mathrm{Cl}(2)$ & $3.0741(17)$ & $\mathrm{Nd}(1)-\mathrm{C}(2)$ & $2.875(3)$ & $\mathrm{Nd}(1)-\mathrm{C}(26)$ & $2.859(3)$ \\
$\mathrm{K}(1)-\mathrm{O}(3)$ & $2.677(5)$ & $\mathrm{Nd}(1)-\mathrm{C}(3)$ & $2.713(3)$ & $\mathrm{Nd}(1)-\mathrm{C}(27)$ & $2.782(3)$ \\
$\mathrm{K}(1)-\mathrm{O}(4)$ & $2.700(7)$ & $\mathrm{Nd}(1)-\mathrm{C}(4)$ & $2.687(3)$ & $\mathrm{Nd}(1)-\mathrm{C}(28)$ & $2.776(3)$ \\
$\mathrm{K}(1)-\mathrm{O}(5)$ & $2.827(16)$ & $\mathrm{Nd}(1)-\mathrm{C}(5)$ & $2.800(3)$ & $\mathrm{Nd}(1)-\mathrm{C}(29)$ & $2.775(3)$ \\
$\mathrm{K}(1)-\mathrm{O}(6)$ & $2.563(7)$ & $\mathrm{C}(1)-\mathrm{C}(2)$ & $1.423(4)$ & $\mathrm{C}(25)-\mathrm{C}(26)$ & $1.430(4)$ \\
$\mathrm{Nd}(1)-\mathrm{Cl}(1)$ & $2.6998(12)$ & $\mathrm{C}(1)-\mathrm{C}(5)$ & $1.412(3)$ & $\mathrm{C}(25)-\mathrm{C}(29)$ & $1.412(4)$ \\
$\mathrm{Nd}(1)-\mathrm{Cl}(2)$ & $2.6891(12)$ & $\mathrm{C}(2)-\mathrm{C}(3)$ & $1.408(4)$ & $\mathrm{C}(26)-\mathrm{C}(27)$ & $1.417(3)$ \\
$\mathrm{Nd}(1)-\mathrm{O}(1)$ & $2.974(2)$ & $\mathrm{C}(3)-\mathrm{C}(4)$ & $1.417(4)$ & $\mathrm{C}(27)-\mathrm{C}(28)$ & $1.417(4)$ \\
$\mathrm{Nd}(1)-\mathrm{O}(2)$ & $4.075(3)$ & $\mathrm{C}(4)-\mathrm{C}(5)$ & $1.410(4)$ & $\mathrm{C}(28)-\mathrm{C}(29)$ & $1.413(4)$ \\
\hline \multicolumn{1}{c|}{$\mathrm{Nd}(1)-\mathrm{Cp}$ centroid } & $\mathrm{C}(1)-\mathrm{C}(6)$ & $1.481(4)$ & $\mathrm{C}(25)-\mathrm{C}(30)$ & $1.475(3)$ \\
$\mathrm{Nd}(1)-\mathrm{C}(1) . . \mathrm{C}(5)$ & $2.5296(15)$ & $\mathrm{C}(2)-\mathrm{C}(12)$ & $1.482(4)$ & $\mathrm{C}(26)-\mathrm{C}(36)$ & $1.476(4)$ \\
$\mathrm{Nd}(1)-\mathrm{C}(25) . . \mathrm{C}(29)$ & $2.5363(16)$ & $\mathrm{C}(4)-\mathrm{C}(18)$ & $1.479(4)$ & $\mathrm{C}(28)-\mathrm{C}(42)$ & $1.481(4)$ \\
\hline $\mathrm{Nd}(1)-\mathrm{Cl}(1)-\mathrm{K}(1)$ & $99.18(4)$ & $\mathrm{Cl}(2)-\mathrm{Nd}(1)-\mathrm{Cl}(1)$ & & $85.85(4)$ \\
$\mathrm{Nd}(1)-\mathrm{Cl}(2)-\mathrm{K}(1)$ & $100.06(4)$ & $\mathrm{Cl}(2)-\mathrm{K}(1)-\mathrm{Cl}(1)$ & & $72.94(4)$ \\
\hline
\end{tabular}

Table S7. Dihedral angles between the $\mathrm{Cp}$ and $\mathrm{Ph}$ planes $\left(^{\circ}\right)$ and deviations of $\mathrm{C}_{\mathrm{ipso}}(\mathrm{Ph})$ atoms from the $\mathrm{Cp}$ plane $(\AA)$ in Nd3.

\begin{tabular}{lllll}
\hline $\mathrm{Cp}$ & $\mathrm{Ph}$ & $\mathrm{Cp}-\mathrm{Ph}$ & $\mathrm{C}_{\mathrm{ipso}}(\mathrm{Ph})$ & Deviation \\
\hline $\mathrm{C}(1) . . \mathrm{C}(5)$ & $\mathrm{C}(6) . . \mathrm{C}(11)$ & $39.08(12)$ & $\mathrm{C}(6)$ & $0.136(4)$ \\
$\mathrm{C}(1) . . \mathrm{C}(5)$ & $\mathrm{C}(12) . . \mathrm{C}(17)$ & $40.35(12)$ & $\mathrm{C}(12)$ & $0.176(4)$ \\
$\mathrm{C}(1) . . \mathrm{C}(5)$ & $\mathrm{C}(18) . . \mathrm{C}(23)$ & $42.37(12)$ & $\mathrm{C}(18)$ & $0.048(4)$ \\
$\mathrm{C}(25) . . \mathrm{C}(29)$ & $\mathrm{C}(30) . . \mathrm{C}(35)$ & $37.01(12)$ & $\mathrm{C}(30)$ & $0.111(4)$ \\
$\mathrm{C}(25) . . \mathrm{C}(29)$ & $\mathrm{C}(36) . . \mathrm{C}(41)$ & $40.44(11)$ & $\mathrm{C}(36)$ & $0.259(4)$ \\
$\mathrm{C}(25) . . \mathrm{C}(29)$ & $\mathrm{C}(42) . . \mathrm{C}(47)$ & $22.34(12)$ & $\mathrm{C}(42)$ & $0.239(4)$ \\
\hline
\end{tabular}

Table S8. Weak hydrogen bonds in $\mathbf{N d 3}\left(\AA,^{\circ}\right)$.

\begin{tabular}{lllll}
\hline $\mathrm{D}-\mathrm{H} \ldots \mathrm{A}$ & $\mathrm{d}(\mathrm{D}-\mathrm{H})$ & $\mathrm{d}(\mathrm{H} \ldots \mathrm{A})$ & $\mathrm{d}(\mathrm{D} \ldots \mathrm{A})$ & $<(\mathrm{DHA})$ \\
\hline $\mathrm{C}(24)-\mathrm{H}(24 \mathrm{~A}) \ldots \mathrm{Cl}(1)$ & 0.98 & 2.79 & $3.507(4)$ & 130.3 \\
$\mathrm{C}\left(499^{\prime}\right)-\mathrm{H}(49 \mathrm{C}) \ldots \mathrm{O}\left(5^{\prime}\right)$ & 0.99 & 2.57 & $3.17(3)$ & 118.7 \\
$\mathrm{C}\left(56^{\prime}\right)-\mathrm{H}(56 \mathrm{D}) \ldots \mathrm{Cl}(2)$ & 0.99 & 2.96 & $3.739(14)$ & 136.1 \\
\hline
\end{tabular}




\section{S3.5. $\left\{\left[\eta^{5}-1,2-\mathrm{Ph}_{2}-4-\left(2-\mathrm{MeOC}_{6} \mathrm{H}_{4}\right) \mathrm{C}_{5} \mathrm{H}_{2}\right]_{2} \mathrm{NdCl}\right\}, \mathrm{Nd} 4$}

The complex $\left\{\left[\eta^{5}-1,2-\mathrm{Ph}_{2}-4-\left(2-\mathrm{MeOC}_{6} \mathrm{H}_{4}\right) \mathrm{C}_{5} \mathrm{H}_{2}\right]_{2} \mathrm{NdCl}\right\}$ lies on a 2-fold proper rotation axis passing through the $\mathrm{Nd}-\mathrm{Cl}$ bond (Fig. S16). The $\mathrm{Nd}^{3+}$ cation $\left(\mathrm{CN}_{\mathrm{Nd}}=9\right)$ is coordinated by $\mathrm{Cl}^{-}$, by $\eta^{5}$ cyclopentadienyl ligands and by both ortho-methoxy groups. The dihedral angle between two Cp planes is $53.62(15)^{\circ}$.

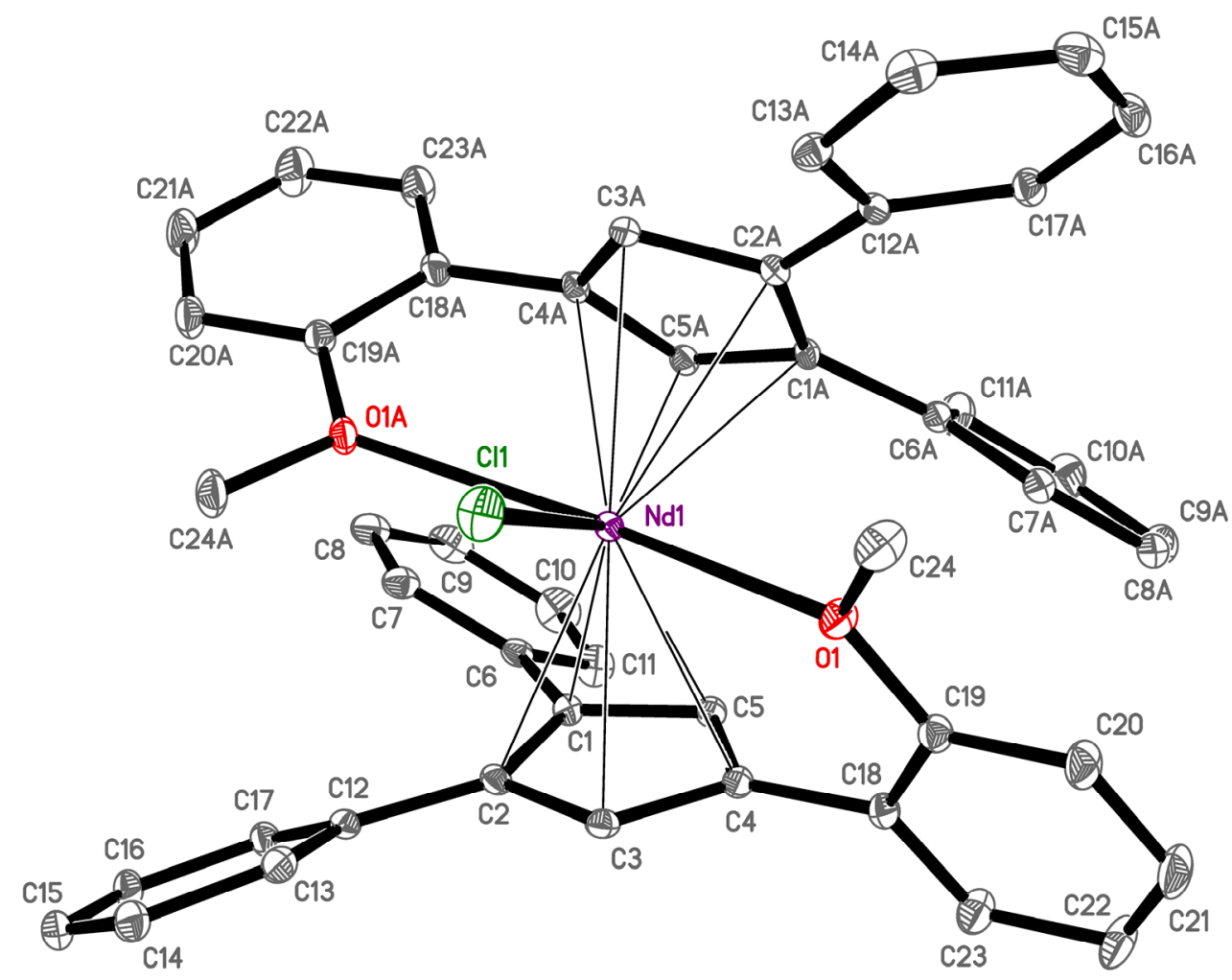

Figure S16. The structure of $\mathbf{N d 4}$. Thermal ellipsoids are set to the $30 \%$ probability level. $\mathrm{H}$ atoms are omitted for clarity. Symmetry code: A $-y+1,-x+1,-z+3 / 2$

Table S9. Selected distances $(\AA)$ and angles $\left(^{\circ}\right)$ in Nd4.

\begin{tabular}{|c|c|c|c|c|c|}
\hline $\mathrm{Nd}(1)-\mathrm{C}(1)$ & $2.800(3)$ & $C(1)-C(2)$ & $1.440(4)$ & $\mathrm{Nd}(1)-\mathrm{O}(1)$ & $2.653(2)$ \\
\hline $\mathrm{Nd}(1)-\mathrm{C}(2)$ & $2.794(3)$ & $C(1)-C(5)$ & $1.422(4)$ & $\mathrm{Nd}(1)-\mathrm{Cl}(1)$ & $2.6578(12)$ \\
\hline $\mathrm{Nd}(1)-\mathrm{C}(3)$ & $2.723(3)$ & $\mathrm{C}(2)-\mathrm{C}(3)$ & $1.427(4)$ & $C(1)-C(6)$ & $1.488(4)$ \\
\hline $\mathrm{Nd}(1)-\mathrm{C}(4)$ & $2.724(3)$ & $C(3)-C(4)$ & $1.407(5)$ & $\mathrm{C}(2)-\mathrm{C}(12)$ & $1.479(5)$ \\
\hline $\mathrm{Nd}(1)-\mathrm{C}(5)$ & $2.750(3)$ & $C(4)-C(5)$ & $1.411(5)$ & $\mathrm{C}(4)-\mathrm{C}(18)$ & $1.482(5)$ \\
\hline \multicolumn{3}{|c|}{$\mathrm{Nd}(1)-\mathrm{C}(1) . . \mathrm{C}(5)$ (centroid) } & $4794(15)$ & & \\
\hline $\mathrm{O}(1)-\mathrm{Nd}(1)-\mathrm{Cl}(1)$ & $77.10(6)$ & \multirow{2}{*}{\multicolumn{2}{|c|}{$C p_{\text {(centroid) }}-\mathrm{Nd}(1)-\mathrm{Cp}_{\text {(centroid) }}$}} & \multicolumn{2}{|c|}{124.9} \\
\hline $\mathrm{O}(1) \# 1-\mathrm{Nd}(1)-\mathrm{O}(1)$ & $154.20(12)$ & & & \multicolumn{2}{|c|}{117.5} \\
\hline
\end{tabular}

Symmetry transformation to generate equivalent atoms: $\# 1-\mathrm{y}+1,-\mathrm{x}+1,-\mathrm{z}+3 / 2$ (a 2-fold rotation axis) 
Table S10. Dihedral angles between the $\mathrm{Cp}$ and $\mathrm{Ph}$ planes $\left(^{\circ}\right)$ and deviations of $\mathrm{C}_{\mathrm{ipso}}(\mathrm{Ph})$ atoms from the $\mathrm{Cp}$ plane $(\AA)$ in Nd4.

\begin{tabular}{lllll}
\hline $\mathrm{Cp}$ & $\mathrm{Ph}$ & $\mathrm{Cp}-\mathrm{Ph}$ & $\mathrm{C}_{\mathrm{ipso}}(\mathrm{Ph})$ & Deviation \\
\hline $\mathrm{C}(1) . . \mathrm{C}(5)$ & $\mathrm{C}(6) . . \mathrm{C}(11)$ & $35.55(17)$ & $\mathrm{C}(6)$ & $0.029(6)$ \\
$\mathrm{C}(1) . . \mathrm{C}(5)$ & $\mathrm{C}(12) . . \mathrm{C}(17)$ & $25.39(16)$ & $\mathrm{C}(12)$ & $0.179(6)$ \\
$\mathrm{C}(1) . . \mathrm{C}(5)$ & $\mathrm{C}(18) . . \mathrm{C}(23)$ & $70.86(14)$ & $\mathrm{C}(18)$ & $0.027(6)$ \\
\hline
\end{tabular}

\section{S4. The studies of the CCTP of ethylene}

\section{S4.1. Oligomerization and derivatization}

\section{Synthesis of $\mathrm{PE}_{2} \mathrm{Mg}$ and PE-MgBHT.}

In a typical experiment, a lanthanide complex (Nd1, Tb1, Nd2 or Nd3) and either (BHT)Mg $(\mathrm{THF}){ }_{2}{ }^{n} \mathrm{Bu}$ or $\mathrm{Mg}^{n} \mathrm{Bu}_{2}$ solution $([\mathrm{Mg}] /[\mathrm{Nd}]=20: 1)$ were dissolved in toluene to reach the required concentration $\left([\mathrm{Nd}]=4 \cdot 10^{-4} \mathrm{M}\right)$. The resulting solution was placed into a glass reactor equipped with a stir bar and degassed on a vacuum line. The reactor was then connected to an ethylene reservoir with a pressure sensor, rapidly placed into a thermostat with a preset polymerization temperature (40 ${ }^{\circ} \mathrm{C}$ ), and exposed to an ethylene pressure of $0.15 \mathrm{MPa}$. The pressure was kept constant in the reactor during oligomerization. The consumption rate of ethylene was measured from the observed drop of pressure in the reservoir. Kinetic measurements were performed at $1 \mathrm{~h}$ or less if the ethylene consumption ceased earlier. The reactor was cooled to room temperature, degassed, and brought into a glove box.

In experiments for obtaining organomagnesium compounds with relatively short PE-chains (average PE-lengths were $\mathrm{C}_{20}$ to $\mathrm{C}_{40}$ based on $M_{n}$ from MS data for corresponding [PE-DMAP]I), a controlled ethylene consumption was used.

For luminescent studies, all solutions were evaporated from an aliquot of a reaction mixture under dynamic vacuum, and the resulting residue was sealed in evacuated quartz tube with outer diameter $4 \mathrm{~mm}$.

\section{Synthesis of PE-I.}

A freshly prepared solution of $\mathrm{I}_{2}$ (2.3 eq. for $\mathrm{PE}_{2} \mathrm{Mg}$ or 3.4 eq. for $\mathrm{PE}-\mathrm{MgBHT}$ ) in absolute THF was dropwise added under argon to an aliquot of a reaction mixture at $0-5{ }^{\circ} \mathrm{C}$. The mixture was stirred under argon for $30 \mathrm{~min}$ at $5{ }^{\circ} \mathrm{C}$ and then overnight at room temperature. The mixture was then washed with methanol, dilute water solutions of $\mathrm{NaHSO}_{3}$ and of $\mathrm{HCl}(<1 \mathrm{M})$. The resulting 
white-off precipitate was filtered off, rinsed with water and a large amount of methanol, and dried under dynamic vacuum to the constant mass.

\section{Synthesis of [PE-DMAP]I.}

The [PE-DMAP]I derivatives were obtained by reaction of PE-I with ca. 2 eq. of 4-(N,Ndimethylamino)pyridine in $o$-dichlorobenzene. The reaction mixture temperature was gradually increased to $80-110^{\circ} \mathrm{C}$, at which the reaction mixture was stirred during $4-24 \mathrm{~h}$ depending on an expected PE-chain length.

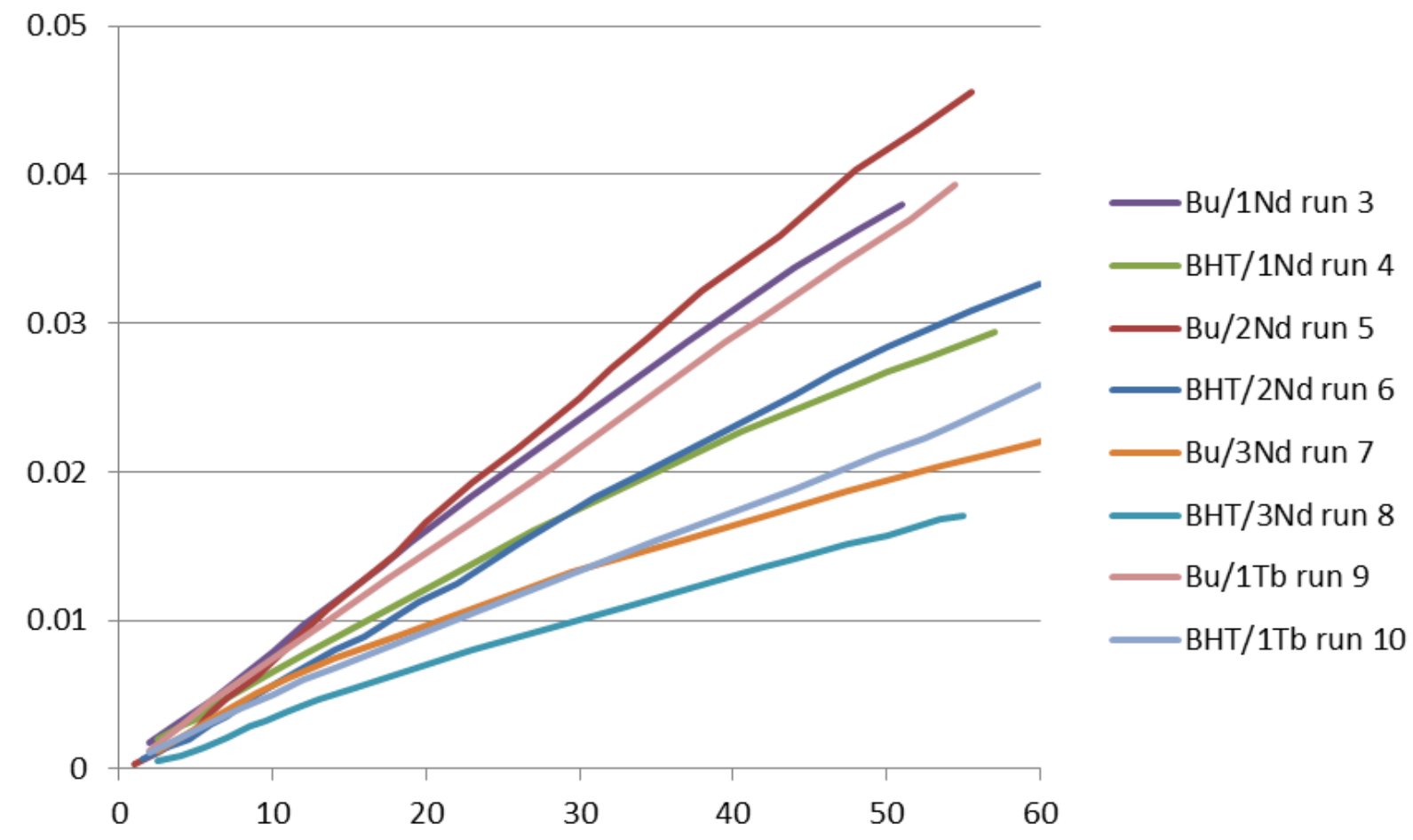

Fig. S17. Ethylene consumption kinetic profiles for Table 1, runs 2-9 (mol of ethylene / $\min$ ).

$51 \mu \mathrm{mol} \mathrm{Nd},[\mathrm{Nd}]=4 \cdot 10^{-4} \mathrm{M}$ 
S4.2. NMR spectra of reaction products

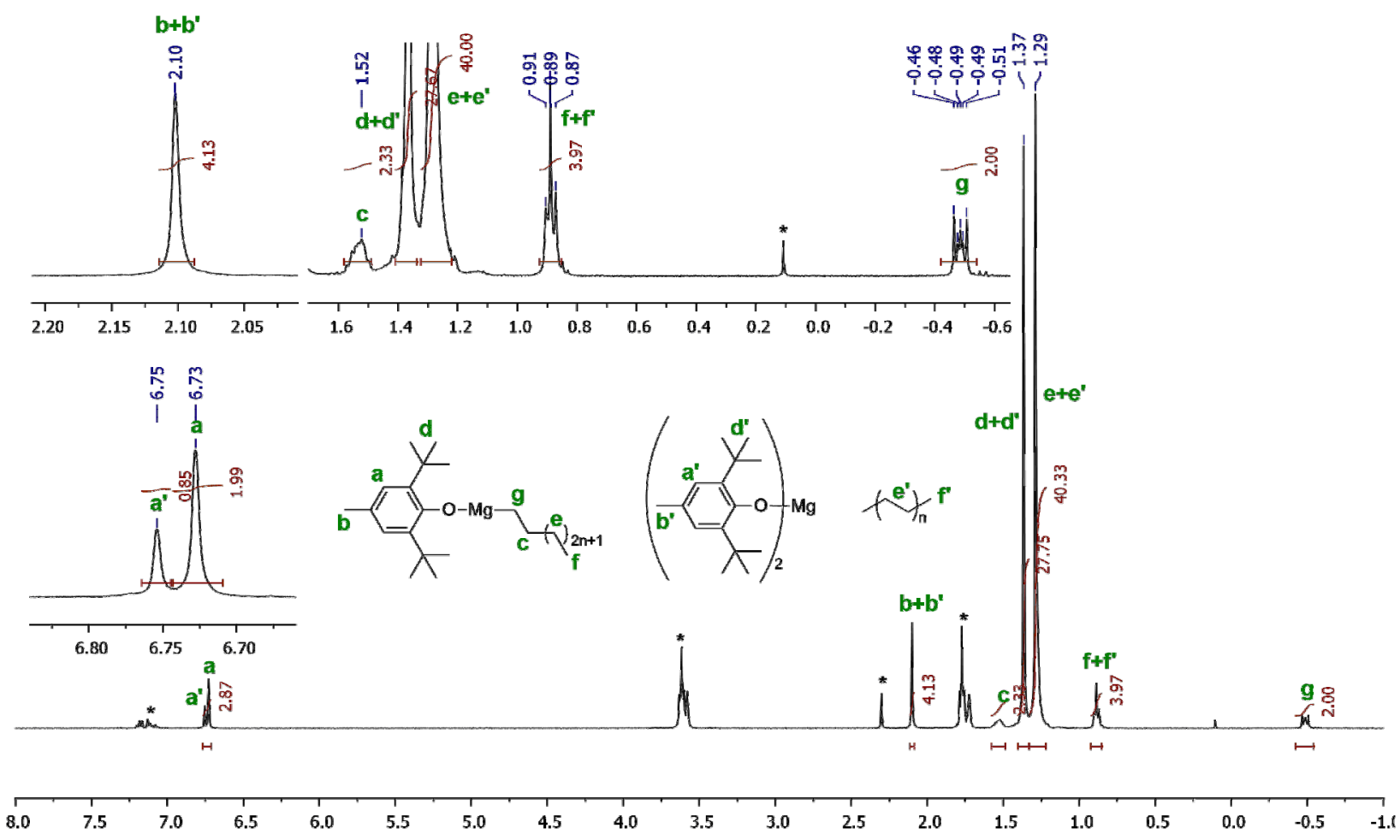

Fig.S18. ${ }^{1} \mathrm{H}$ NMR spectrum $\left(400 \mathrm{MHz}\right.$, THF- $\left.\mathrm{d}_{8}, 20^{\circ} \mathrm{C}\right)$ of the reaction mixture. $\mathrm{Nd} 1 /(\mathrm{BHT}) \mathrm{Mg}(\mathrm{THF})_{2}{ }^{{ }^{n} \mathrm{Bu}}$ catalyst; $[\mathrm{Nd}] /[\mathrm{Mg}]=1: 20$. The average $\mathrm{PE}$ length is $\mathrm{C}_{20}$. An approximate $(\mathrm{BHT}) \mathrm{Mg}-\mathrm{PE} /(\mathrm{BHT})_{2} \mathrm{Mg} / \mathrm{PE}$ ratio 73:15:12.
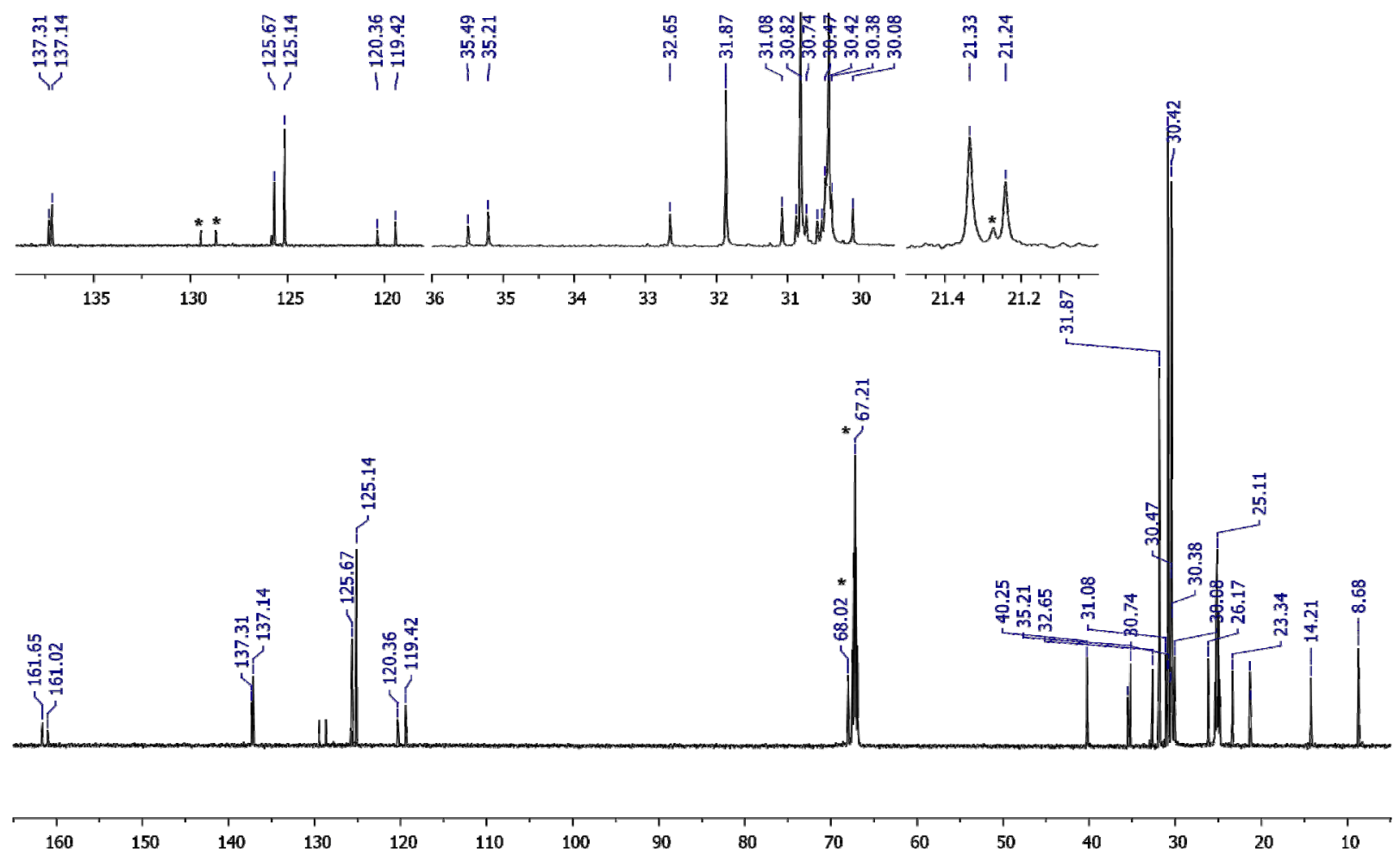

Fig.S19. ${ }^{13} \mathrm{C}\left\{{ }^{1} \mathrm{H}\right\}$ NMR spectrum $\left(101 \mathrm{MHz}, \mathrm{THF}-\mathrm{d}_{8}, 20^{\circ} \mathrm{C}\right)$ of the reaction mixture, $\mathrm{Nd} 1 /(\mathrm{BHT}) \mathrm{Mg}(\mathrm{THF}){ }_{2}{ }^{n} \mathrm{Bu}$ catalyst; $[\mathrm{Nd}] /[\mathrm{Mg}]=1: 20$. 

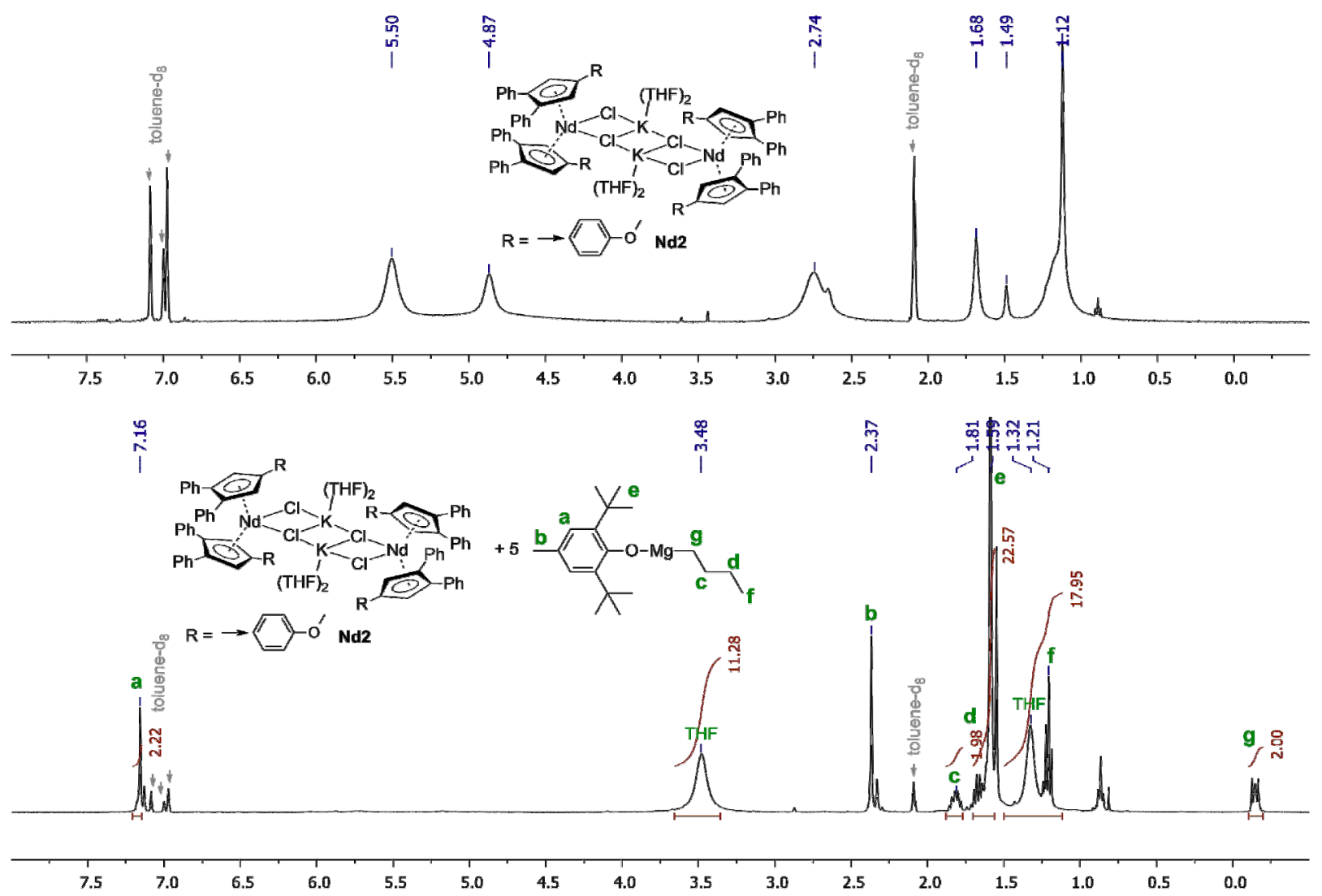

Fig. S20. ${ }^{1} \mathrm{H}$ NMR spectra (toluene- $\mathrm{d}_{8}$ ) of $\mathbf{N d 2}$ (top) and $\mathbf{N d 2}+5$ eq. (BHT)Mg ${ }^{n}$ Bu reaction mixture (bottom). 


\section{S4.3. Characterization of [PE-DMAP]I}

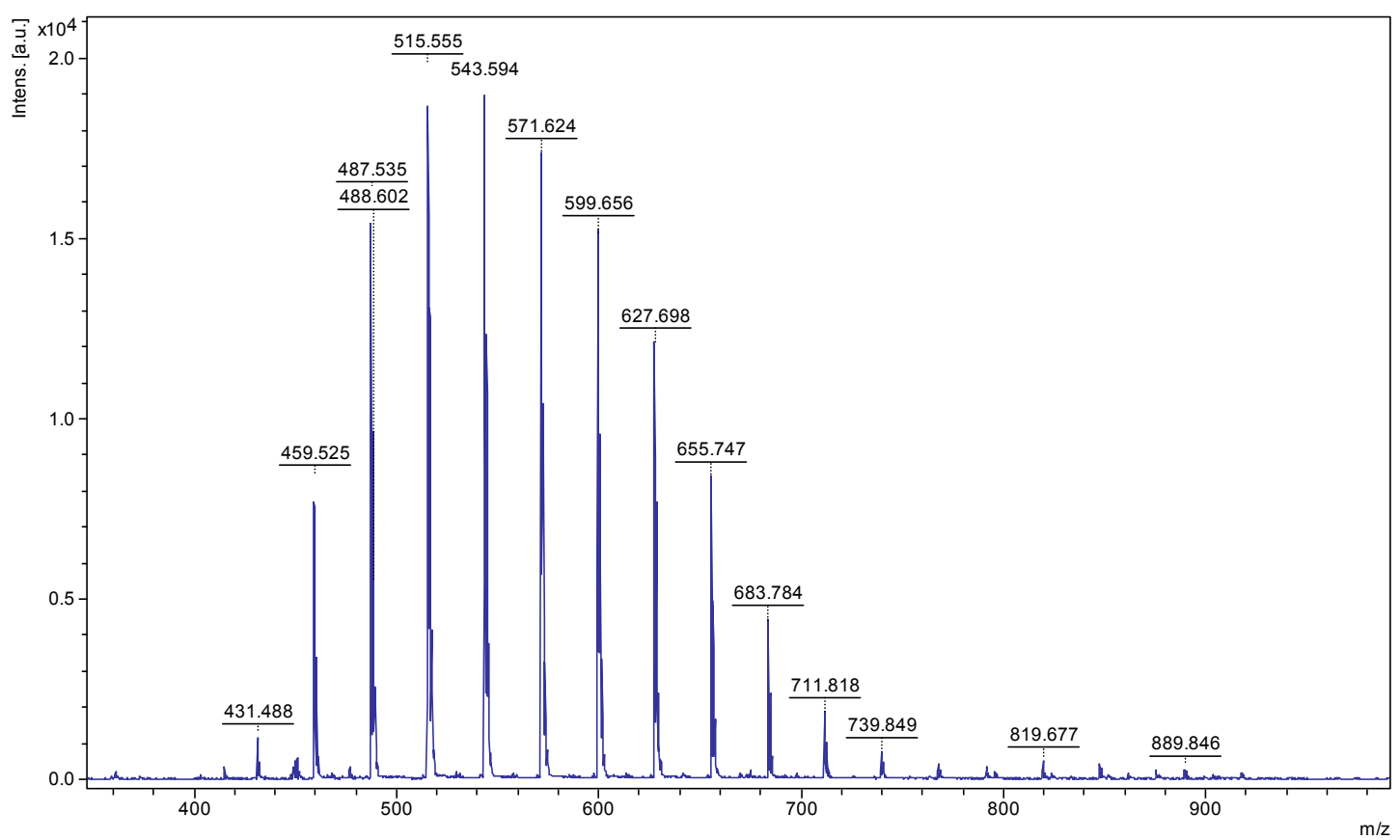

Fig. S21. A typical mass-spectrum example for [PE-DMAP]I with a short PE chain. $M_{n}=565\left(C_{32}\right), M_{w}=573$, $\mathrm{PD}=1.014$. The $\mathrm{PE}$ chain range: $\mathrm{C}_{22}$ to $\mathrm{C}_{44}\left((\mathrm{BHT}) \mathrm{Mg}^{n} \mathrm{Bu} / \mathrm{Nd} 1\right.$ system, $40{ }^{\circ} \mathrm{C}, 0.15 \mathrm{MPa}$, short time of ethylene consumption). See Figs. S18 and S19.

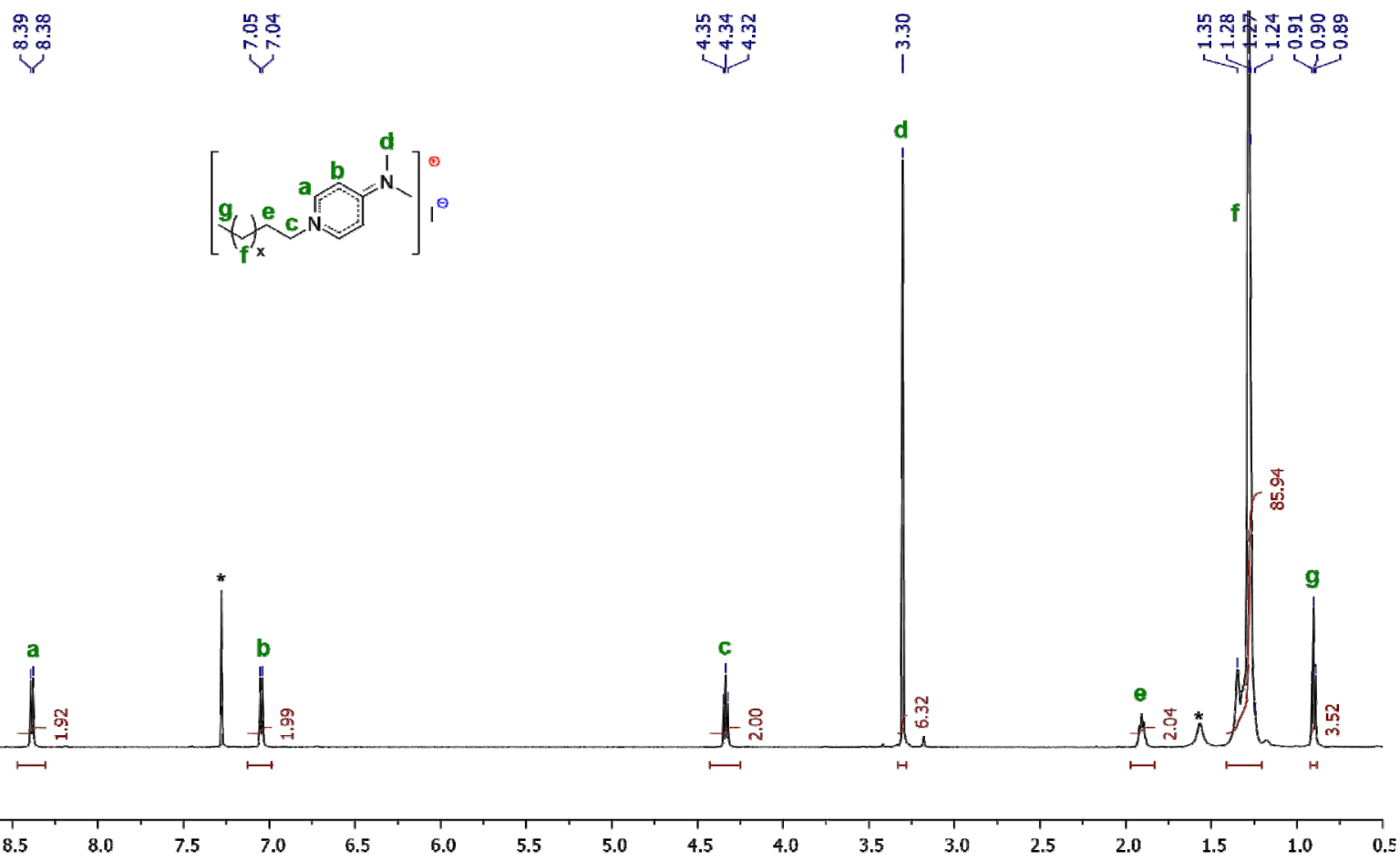

Fig. S22. An example of ${ }^{1} \mathrm{H}$ NMR spectrum for [PE-DMAP]I bearing a short PE-chain $\left(\mathrm{CDCl}_{3}\right)$. The averaged PE length is $\mathrm{C}_{40}$ by NMR. The functionalization degree is $94 \%$ by NMR. The spectrum corresponds to the sample above (see Figs. 2, S18 and S19). 


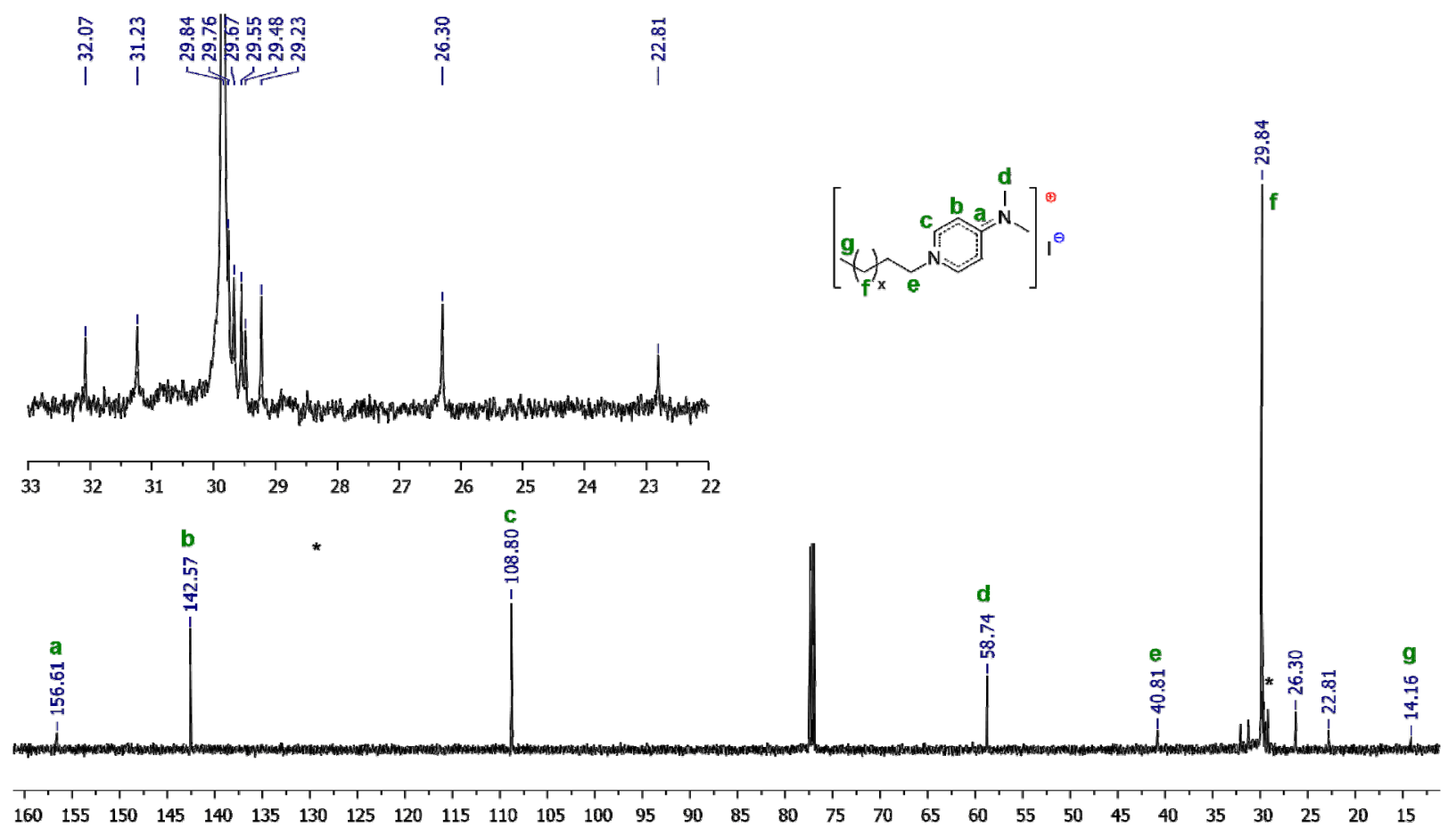

Fig. S23. An example of NMR ${ }^{13} C\left\{{ }^{1} \mathrm{H}\right\}$ spectrum for [PE-DMAP]I bearing a short PE-chain $\left(\mathrm{CDCl}_{3}\right)$. The spectrum corresponds to the sample above (see Figs. S18, S19).
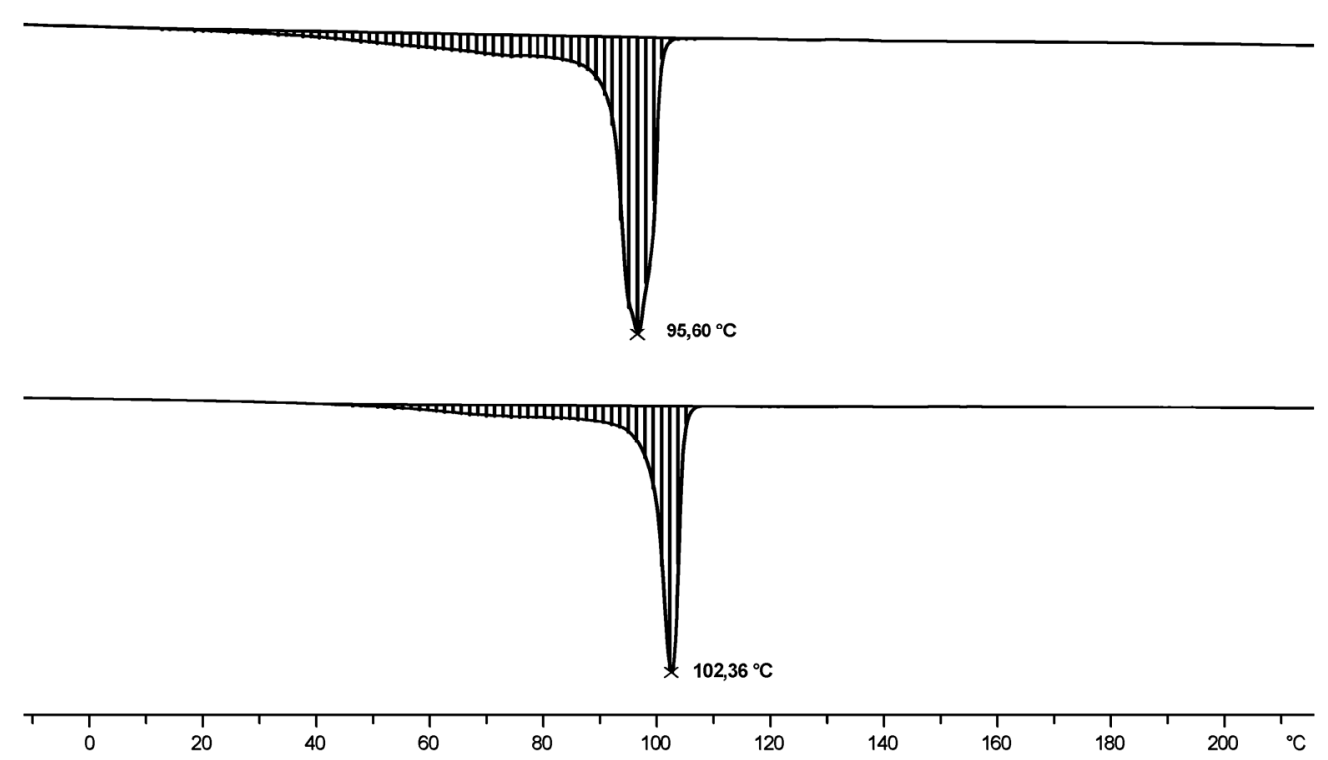

Fig. S24. Typical examples of DSC curves ( $2^{\text {nd }}$ heating) for [PE-DMAP]I bearing a short PE-chain and obtained from (BHT)Mb ${ }^{n} \mathrm{Bu} / \mathrm{Nd1}$ system (top; corresponds to the sample above; Figs. S18-S19) and $\mathrm{Mg}^{n} \mathrm{Bu}_{2} / \mathrm{Nd} 3$ system (bottom; corresponds to run 7 in Table 1). 


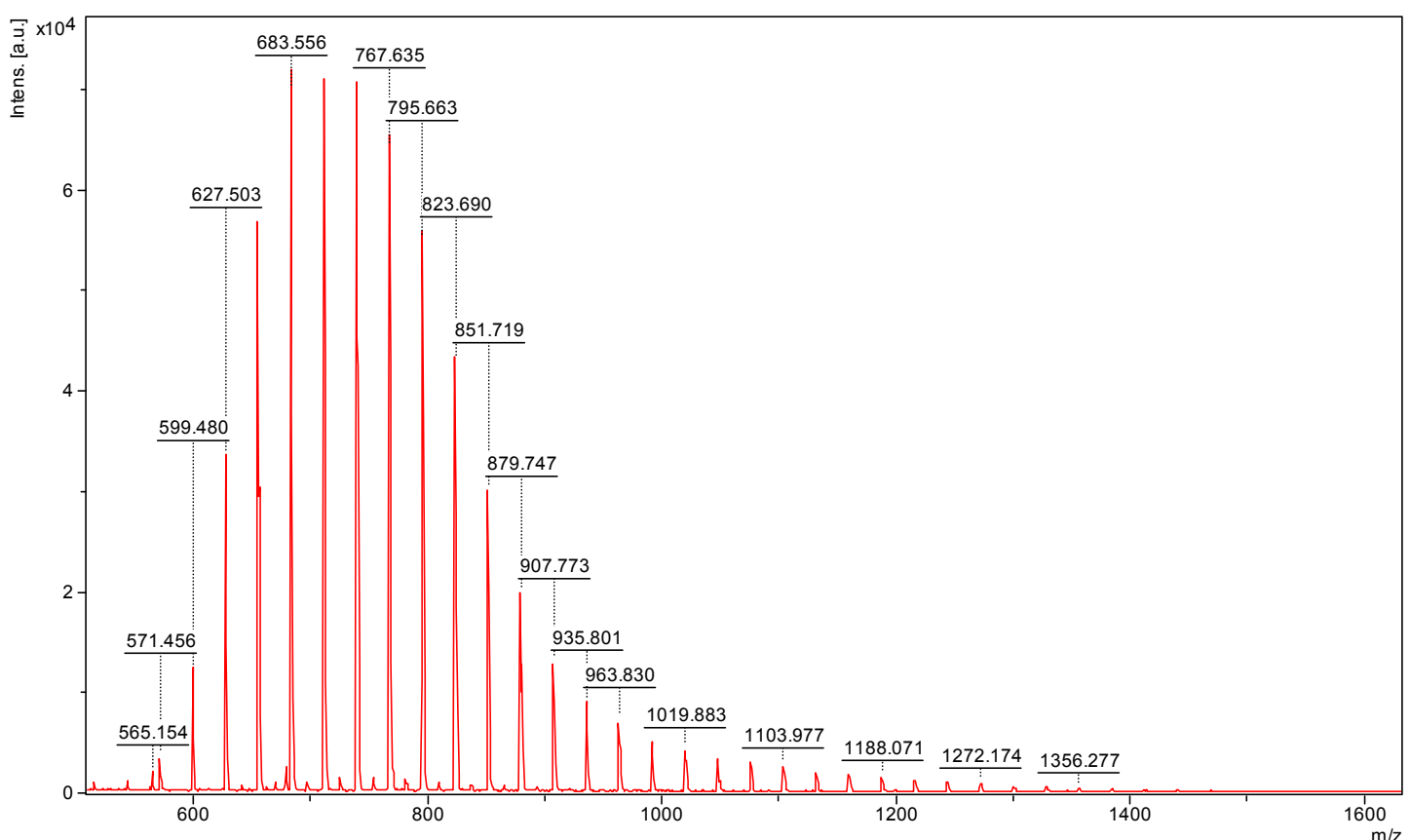

Fig. S25. A typical mass-spectrum example for [PE-DMAP]I with a longer PE chain. $M_{n}=768\left(C_{46}\right), M_{w}=786$, $P D=1.023$. The $P E$ chain range: $C_{32}$ to $C_{76}$ (Table 1, run 5).

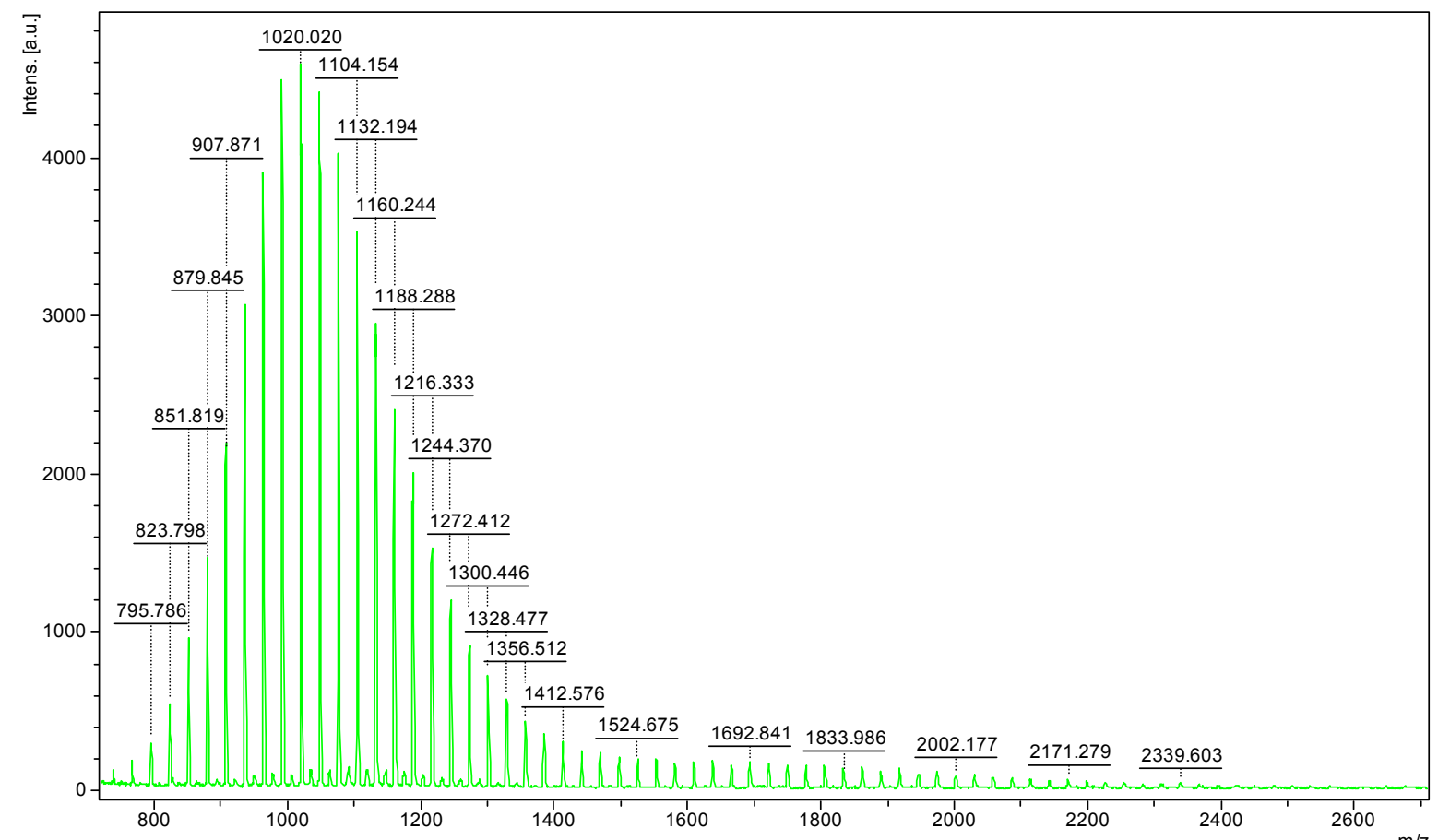

Fig. S26. A typical mass-spectrum example for [PE-DMAP]I with a long PE chain. $M_{n}=1403\left(C_{92}\right), M_{w}=1520$, $\mathrm{PD}=1.083$. (Table 1, run 6). 


\section{Nd}

$123,66^{\circ} \mathrm{C}$

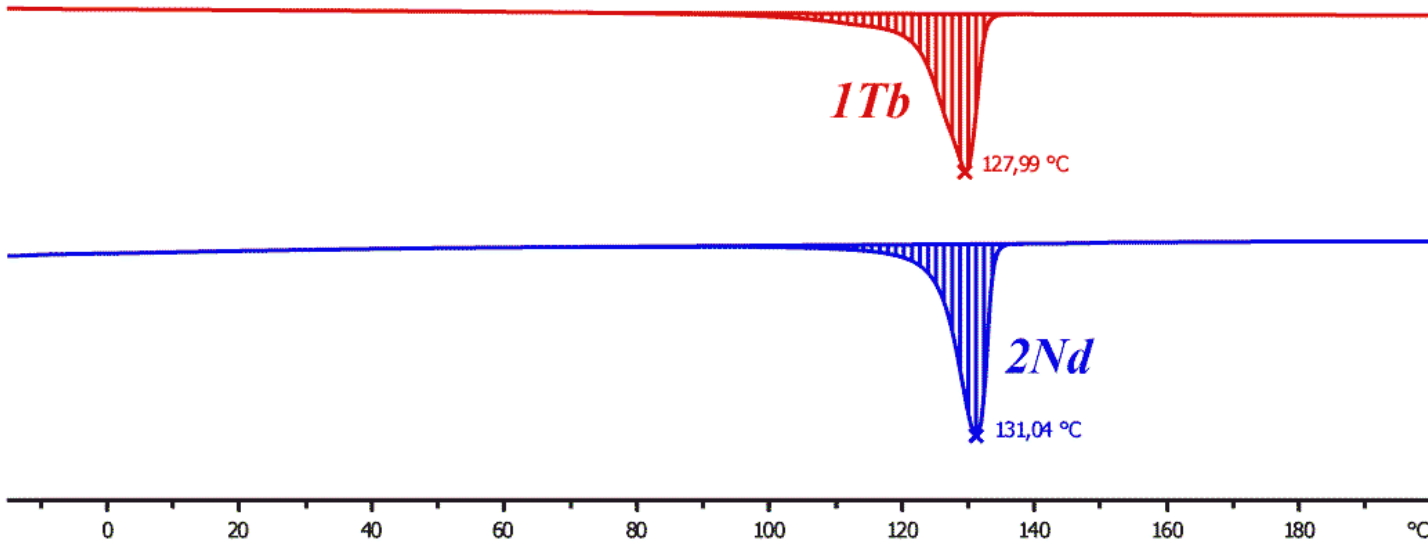

Fig. S27. DSC curves ( ${ }^{\text {nd }}$ heating) for [PE-DMAP]I bearing long PE-chains and obtained by using Nd1 (top), Nd2 (middle) and Tb1 (bottom) precatalysts and (BHT) $\mathrm{Mg}^{n} \mathrm{Bu}$. See Table 1 runs 4, 10 and 6, respectively.

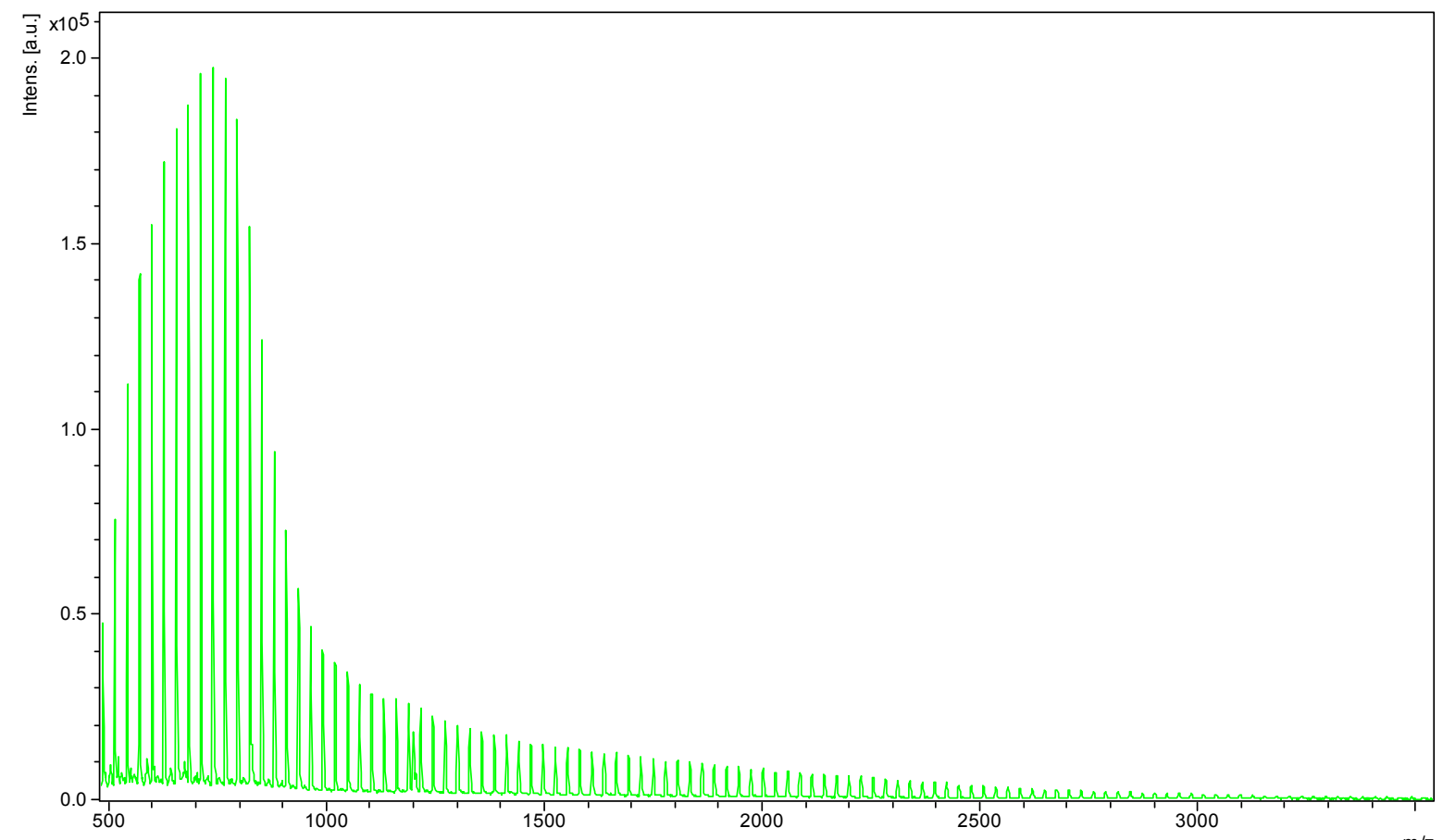

Fig. S28. Mass-spectrum for [PE-DMAP]I formed from (BHT)Mg(PE) obtained at $80^{\circ} \mathrm{C}\left((\mathrm{BHT}) \mathrm{Mg}{ }^{n} \mathrm{Bu} / \mathrm{Nd2}\right.$ system). $M_{n}=1100\left(C_{70}\right), M_{w}=1387, P D=1.260$ (Table 1 , run 12). 


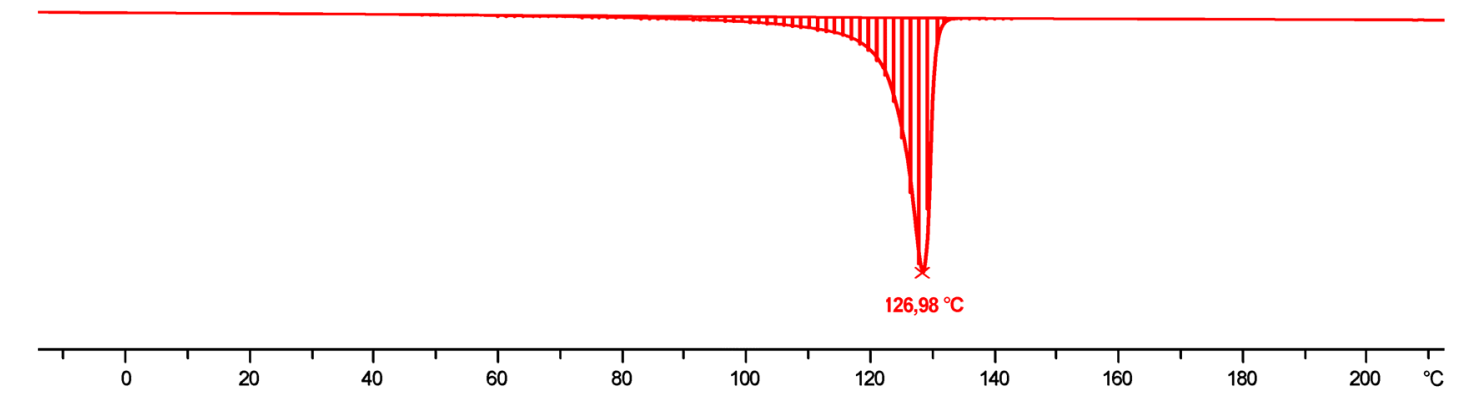

Fig. S29. DSC curve $\left(2^{\text {nd }} \text { heating) for the [PE-DMAP }\right]^{+}{ }^{-}$sample above (Table 1 , run 12$)$.



Fig. S30. A mass-spectrum example for [PE-DMAP]I prepared from PE-Mg-BHT obtained at higher ethylene pressure (0.3 $\mathrm{MPa}, 2 \mathrm{~h}, 40^{\circ} \mathrm{C}$, $(\mathrm{BHT}) \mathrm{Mg}^{n} \mathrm{Bu} / \mathrm{Nd2}$ system). $\mathrm{M}_{\mathrm{n}}=1651$ (average $\mathrm{C}_{109}$ ), $\mathrm{M}_{\mathrm{w}}=1776, \mathrm{PD}=1.075$ (See Table 1 run 14).

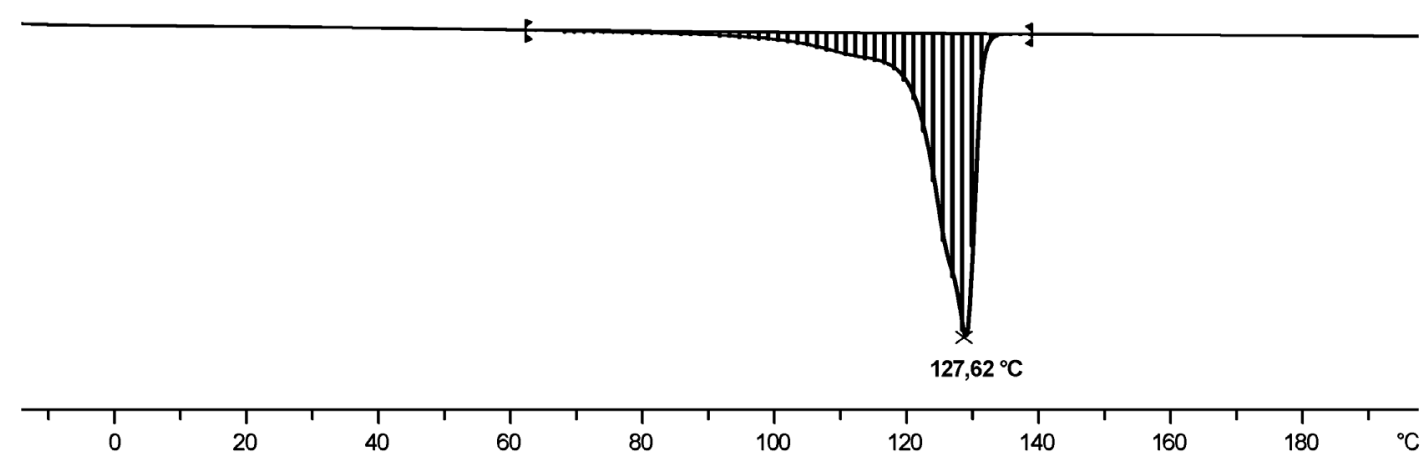

Fig. S31. The DSC curve ( $2^{\text {nd }}$ heating) for the sample of [PE-DMAP]I above (Table 1 run 14). 


\section{S5. Optical measurements}

Steady-state luminescence and excitation measurements in the visible region were performed with a Horiba-Jobin-Yvon-Spex Fluorolog FL 3-22 spectrometer, which had a $450 \mathrm{~W}$ xenon lamp as the excitation source and a R-928 photomultiplier. The technique involved the use of a specially designed, sealed quartz cuvette for manipulation of the air-sensitive compounds during luminescence measurements. Luminescence lanthanide lifetimes $(\tau)$ were measured at least three times by monitoring the decay at the maxima of the emission spectra. The single or biexponential decays were analyzed with Origin ${ }^{\circledR} 8.1$.

\section{S6. DFT calculations}

Quantum chemistry computations were performed in the Gaussian09 program. ${ }^{15}$ The optimization was calculated with the hybrid PBE0 functional ${ }^{16}$ and large-core energy-adjusted RECPs for Tb, developed by the Stuttgart and Dresden groups, along with the accompanying basis set ECP54MWB to describe the valence electron density. ${ }^{17,18}$ Large-core energy-adjusted RECPs for $\mathrm{Tb}$ put $5 \mathrm{~s}, 5 \mathrm{p}, 6 \mathrm{~d}$, and $6 \mathrm{~s}$ shells in the valence space, whereas $4 \mathrm{f}$ electrons belonged to the core pseudopotentials. For other atoms, a 6-311+g* basis set was employed. Tight SCF convergence and standard optimization convergence criteria along with ultrafine grids were always used during the calculations. The geometry of $\left[\left(\mathrm{C}_{5} \mathrm{H}_{2} \mathrm{Ph}_{3}\right)_{2} \mathrm{~Tb}\left(\mu-{ }^{n} \mathrm{Bu}\right)_{2} \mathrm{Mg}\left(\mu-{ }^{n} \mathrm{Bu}\right)_{2} \mathrm{Mg}{ }^{n} \mathrm{Bu}\right]$ was optimized using the very tight optimization criteria and empirical dispersion corrections on the total energy ${ }^{19}$ with the Becke-Johnson damping (D3). ${ }^{20}$

\section{References}

(1) Brown, T. L.; Dickerhoof, D. W.; Bafus, D. A.; Morgan, G. L. Method for Removal of Oxygen from Inert Atmosphere Enclosures. Rev .Sci. Inst. 1962, 33, 491-492.

(2) Horvath, B.; Möseler , R.; Horvath, E. G.; Krauss, H. L. Über Oberflächenverbindungen von Übergangsmetallen. XI. Darstellung und Eigenschaften von Koordinativ ungesättigten Mangan(II)Oberflächenverbindungen. Z. Anorg. Allg. Chem. 1975, 418, 1-16.

(3) Zhang, X.; Ye, J.; Xu, L.; Yang, L.; Deng, D.; Ning, G. Synthesis, crystal Structures and aggregation-induced emission enhancement of aryl-substituted cyclopentadiene derivatives. Journal of Luminescence 2013, 139, 28-34 
(4) Edelmann, F. T.; Poremba, P. Inorganic Compounds and Important Starting Materials of the Lanthanide Elements. In Synthetic Methods of Organometallic and Inorganic Chemistry (Herman/Brauer). Lanthanides and Actinides., Ed. by Edelmann, F.T.; Herrmann, W.A., Verlag: Stuttgart, Germany, 1997; Vol. 6, 34-35.

(5) Nifant'ev, I. E.; Shlyakhtin, A. V.; Tavtorkin, A. N.; Ivchenko, P. V.; Borisov, R. S.; Churakov, A. V. Monomeric and dimeric magnesium mono-BHT complexes as effective ROP catalysts. Catal. Commun. 2016, 87, 106-111.

(6) Minyaev, M. E.; Vinogradov, A. A.; Roitershtein, D. M.; Borisov, R. S.; Ananyev, I. V.; Churakov, A. V.; Nifant'ev, I. E. Catalytic activity of phenyl substituted cyclopentadienyl neodymium complexes in the ethylene oligomerization process. J. Organomet. Chem. 2016, 818, 128-136.

(7) Schlosser, M.; Hartmann, J. Transmetalation and Double Metal Exchange: A Convenient Route to Organolithium Compounds of the Benzyl and Allyl Type. Angew. Chem., Int. Ed. Eng. 1973, 12, 508-510.

(8) Roitershtein, D. M.; Puntus, L. N.; Vinogradov, A. A.; Lyssenko, K. A.; Minyaev, M. E.; Dobrokhodov, M. D.; Taidakov, I. V.; Varaksina, E. A.; Churakov, A. V.; Nifant'ev, I. E. Polyphenylcyclopentadienyl Ligands as an Effective Light-Harvesting $\pi$-Bonded Antenna for Lanthanide +3 Ions. Inorg. Chem. 2018, 57, 10199-10213

(9) Ellis, J. E.; Minyaev, M. E.; Nifant'ev, I. E.; Churakov, A. V. Scandium complexes with the tetraphenylethylene and anthracene dianions. Acta Cryst. 2018, C74, 769-781.

(10) Bruker. APEXII, Bruker AXS Inc.: Madison, Wisconsin, USA, 2008.

(11) Sheldrick, G. M. A short history of SHELX. Acta Cryst. 2008, A64, 112-122.

(12) Sheldrick, G. M. Crystal structure refinement with SHELXL. Acta Cryst. 2015, C71, 3-8.

(13) Sheldrick, G. M. SHELXT - Integrated space-group and crystal-structure determination. Acta Cryst. 2015, A71, 3-8.

(14) Roitershtein, D. M.; Puntus, L. N.; Vinogradov, A. A.; Lyssenko, K. A.; Minyaev, M. E.; Dobrokhodov, M. D.; Taidakov, I. V.; Varaksina, E. A.; Churakov, A. V.; Nifant'ev, I. E. Polyphenylcyclopentadienyl Ligands as an Effective Light-Harvesting $\pi$-Bonded Antenna for Lanthanide $^{+3}$ Ions. Inorg. Chem. 2018, 57, 10199-10213.

(15) Frisch, M.; Trucks, G.; Schlegel, H.; Scuseria, G.; Robb, M.; Cheeseman, J.; Scalmani, G.; Barone, V.; Petersson, G.; Nakatsuji, H.; Li, X.; Caricato, M.; Marenich, A.; Bloino, J.; Janesko, B.; Gomperts, R.; Mennucci, B.; Hratchian, H.; Ortiz, J.; Izmaylov, A.; Sonnenberg, J.; WilliamsYoung, D.; Ding, F.; Lipparini, F.; Egidi, F.; Goings, J.; Peng, B.; Petrone, A.; Henderson, T.; Ranasinghe, D.; Zakrzewski, V.; Gao, J.; Rega, N.; Zheng, G.; Liang, W.; Hada, M.; Ehara, M.; 
Toyota, K.; Fukuda, R.; Hasegawa, J.; Ishida, M.; Nakajima, T.; Honda, Y.; Kitao, O.; Nakai, H.; Vreven, T.; Throssell, K.; Montgomery, J.; Peralta, J.; Ogliaro, F.; Bearpark, M.; Heyd, J.; Brothers, E.; Kudin, K.; Staroverov, V.; Keith, T.; Kobayashi, R.; Normand, J.; Raghavachari, K.; Rendell, A.; Burant, J.; Iyengar, S.; Tomasi, J.; Cossi, M.; Millam, J.; Klene, M.; Adamo, C.; Cammi, R.; Ochterski, J.; Martin, R.; Morokuma, K.; Farkas, O.; Foresman, J.; Fox, D. Gaussian 09; Gaussian, Inc., Wallingford CT, 2016.

(16) Perdew, J.; Ernzerhof, M.; Burke, K. Rationale for mixing exact exchange with density functional approximations. J. Chem. Phys. 1996, 105, 9982-9985.

(17) Dolg, M.; Stoll, H.; Savin, A.; Preuss, H. Energy-adjusted pseudopotentials for the rare earth elements. Theor. Chim. Acta 1989, 75, 173-194.

(18) Dolg, M.; Stoll, H.; Preuss, H. A combination of quasirelativistic pseudopotential and ligand field calculations for lanthanoid compounds.

(19) Grimme, S.; Antony, J.; Ehrlich S., Krieg H. A consistent and accurate ab initio parametrization of density functional dispersion correction (DFT-D) for the 94 elements H-Pu. J. Chem. Phys., 2010, 132, 154104-1--154104-19.

(20) Grimme, S.; Ehrlich, S.; Goerigk, L. Effect of the damping function in dispersion corrected density functional theory. J. Comp. Chem., 2011, 32, 1456-1465. 